\title{
The squamous cell carcinoma antigen/SERPINB3 protects cervical cancer cells from chemoradiation by preventing lysoptosis
}

\section{Songyan Wang}

Washington University School of Medicine

\section{Cliff Luke}

Washington University School of Medicine

Stephen Pak

Washington University School of Medicine

Victoria Shi

Washington University School of Medicine

LiYun Chen

Washington University School of Medicine

Jonathan Moore

Washington University School of Medicine

Arlise Andress

Washington University in St. Louis

Huang Yi

Washington University School of Medicine https://orcid.org/0000-0002-2867-1477

Marina Platik

Washington University School of Medicine

Anthony Apicelli

Washington University School of Medicine

Julie Schwarz

Washington University School of Medicine

Perry Grigsby

Washington University School of Medicine

Gary Silverman

Washington University School of Medicine

Stephanie Markovina ( $\nabla$ smarkovina@wustl.edu )

Washington University School of Medicine https://orcid.org/0000-0002-1139-5533 
Keywords: SERPINB3, cervical cancer, lysoptosis, squamous cell carcinoma antigen

Posted Date: February 19th, 2021

DOI: https://doi.org/10.21203/rs.3.rs-228819/v1

License: (c) (1) This work is licensed under a Creative Commons Attribution 4.0 International License. Read Full License

Version of Record: A version of this preprint was published at Communications Biology on January 12th, 2022. See the published version at https://doi.org/10.1038/s42003-021-02893-6. 


\section{Abstract}

The endogenous lysosomal cysteine protease inhibitor SERPINB3 (squamous cell carcinoma antigen 1, SCCA1) is elevated in patients with cervical cancer and other malignancies. High serum SERPINB3 is prognostic for recurrence and death following chemoradiation therapy (CRT). Cervical cancer cells genetically lacking SERPINB3 are more sensitive to ionizing radiation (IR), suggesting this protease inhibitor plays a role in therapeutic response. Here we demonstrate that SERPINB3-deficient cells have enhanced sensitivity to IR-induced cell death. Knock out of SERPINB3 sensitizes cells to a greater extent than cisplatin, the current standard of care. IR in SERPINB3 deficient cervical carcinoma cells induces cell death morphologically consistent with necrosis, with biochemical and cellular features of lysoptosis. Moreover, rescue with wild-type SERPINB3 or a reactive site loop mutant indicates that protease inhibitory activity is required to protect cervical tumor cells from radiation-induced death. These data suggest targeting of SERPINB3 and lysoptosis to treat radioresistant cervical cancers.

\section{Introduction}

Cervical cancer remains a leading cause of cancer death for women worldwide ${ }^{1}$. Despite efforts to improve screening and human papilloma-virus (HPV) vaccination rates, overall mortality from the disease has not changed substantially in the last several decades ${ }^{1,2}$. Definitive chemoradiation is the standard of care for most women with cervical cancer, but is associated with recurrence rates as high as $30-70 \% 3,4$, implying that many cervical cancers display inherent or adaptive cellular resistance to these therapies. For women who experience recurrent disease following CRT, less than $5 \%$ of women survive beyond five years $^{3}$. Thus, there is an unmet need to understand of the molecular mechanisms of resistance to radiation and chemotherapy in cervical cancer, with the goal of developing novel therapies for both upfront and salvage therapy.

We previously demonstrated that elevated serum squamous cell carcinoma antigen (SCCA) is independently prognostic for recurrence and survival following definitive radiation therapy for cervical cancer ${ }^{5,6}$. Moreover, we found that failure of serum SCCA to normalize by the fourth week of treatment was an early indicator of failed response to therapy as indicated by positive post-therapy FDG-PET, and increased recurrence and death ${ }^{6}$. Clinical serum SCCA assays measure levels of SERPINB3 and SERPINB4 proteins, also known as SCCA1 and SCCA2, respectively. These proteins share high homology, and diverge in amino acid sequence primarily within the carboxy-terminal reactive site loop (RSL), which serves as a pseudo-substrate for specific proteases ${ }^{7}$. Binding and cleavage of the RSL by a target protease results in a rapid conformational change in the SERPIN, preventing further processive proteolysis of the SERPIN and results in a covalently bound SERPIN-protease complex that is ultimately degraded ${ }^{8}$.

SERPINB3 is an intracellular cysteine protease inhibitor that is upregulated in many autoimmune diseases and cancer ${ }^{5,11-16}$. The Caenorhabditis elegans (C. elegans) homologue of SERPINB3, SRP-6, 
protects against lysosomal damage and organismal death by inhibiting cysteine protease activity induced by diverse stressors including hypotonic saline, hydrogen peroxide, DNA-damage and reactive oxygen species ${ }^{17}$. This phenotype can be rescued in the srp- 6 null animal by driving expression of wildtype SRP-6, but not RSL-mutant SRP-6, suggesting that inhibition of cysteine proteases is necessary for cytoprotection. SERPINB4 demonstrates specificity for chymotrypsin-like serine proteases in vitro ${ }^{9}$, and has been shown to inhibit granzyme M-induced cell death when overexpressed in HeLa cells, but does not have other well-described cellular functions ${ }^{10}$.

While high levels of SERPINB3 in the serum is associated with poor response to RT in patients with cervical cancer, it is not clear if SERPINB3 impacts radiation response on a cellular level. We hypothesized that SERPINB3 serves as a radioprotective factor in cervical cancer cells. Indeed we found that cervical cancer cell lines engineered by CRISPR-Cas9-mediated gene editing of the SERPINB3 locus resulting in knock-out (B3-KO) were significantly more sensitive to ionizing radiation in vitro compared to isogenic control cells, as determined by clonogenic cell survival assay ${ }^{11}$. The molecular mechanism of protection against radiation is unknown. Murakami et al found that transient transfection of vector-driven SERPINB3 into 293T human embryonic kidney cells resulted in higher survival as measured by MTT assay up to 36 hours after treatment with radiation ${ }^{18}$. Examination of caspases important for apoptosis demonstrated decreased levels of caspase 9 cleavage and caspase 3 activity in cells transfected with SERPINB3. Based on these findings the authors concluded that SERPINB3 suppresses radiation-induced apoptosis. While the regulation of caspases is important in apoptotic cell death pathways, exogenous stressors, such as irradiation, often induce multiple cellular death pathways within a population of cells. Additionally, radiation does not engage apoptosis effectively in most solid tumors due to common dysregulation of pro-apoptotic pathways ${ }^{19}$. Thus, precise molecular mechanism of protection by SERPINB3 against radiation remains unknown.

In the current study, we found that the mechanism of SERPINB3-mediated radioprotection in cervical cancer cells is inhibition of cell death. Moreover, we identified the mode of cell death induced by radiation in SERPINB3-KO cells as lysoptosis, a regulated lysosome-mediated necrosis pathway, with little to no evidence of apoptotic cell death, necroptosis, pyroptosis or ferroptosis. Additionally, the SERPINB3 RSL is required for protection against radiation, further supporting the mechanism of lysosomal protease inhibition. To our knowledge, this is the first report that SERPINB3 protects cancer cells against radiationinduced necrosis, and does so in a lysosomal cysteine protease inhibitory manner. Moreover, we present the first evidence that ionizing radiation induces a lysosome-mediated cell death pathway in cancer cells, particularly when a protective factor is eliminated, suggesting a potential vulnerability for targeting radioresistant cancers.

\section{Results}

\section{SERPINB3-KO cells are more sensitive to radiation and cisplatin-induced cell death}


For these studies, a panel of cervical cancer cell lines was selected to provide the most clinically relevant models. Two cell lines with high levels of SERPINB3 (SW756 and HT3) and two with low to undetectable levels of SERPINB3 ( $\mathrm{SiHa}$ and C33A), had varying sensitivity to radiation as determined by clonogenic cell survival, with the cell lines expressing SERPINB3 more radioresistant than those without SERPINB3 (Supplemental Fig. 1A, B). Importantly, SW756 with a single integrated copy of the HPV type 18 and SiHa with an integrated copy of HPV type 16, were selected to represent commonly HPV-associated cancers. Conversely, HT3 and C33A cell lines, which do not have HPV DNA, were selected to represent the approximately $10 \%$ of HPV negative cervical cancers (Supplemental Fig. 1A, B). Patients with HPV negative cervical cancers in some clinical series have worse outcomes following chemoradiation ${ }^{20}$, and in the case of head and neck squamous cell carcinoma, these cancers are more likely to be resistant to radiation treatment ${ }^{21,22}$. Thus, we felt it important to understand the impact of SERPINB3-KO on tumor cells of both HPV-positive and HPV-negative origins.

To determine if SERPINB3 protects cells against stress-induced death through a conserved mechanism similar to srp-6, we first quantified cell death by exclusion of Sytox ${ }^{\mathrm{TM}}$ nucleic acid stain in cells treated with sham or increasing doses of radiation over a time course of several days. We found that B3-KO in both HT3 and SW756 cervical cancer cells had significantly higher percent cell death at every dose and every time point following treatment with IR, up to a $20 \%$ absolute difference in the SW756 background, and $50 \%$ in the HT3 background (Fig. 1A-C). We then compared the effect of single gene knock out of SERPINB3 on radiosensitivity with or without cisplatin chemotherapy, the radiosensitizing agent used as standard of care for the treatment of patients with cervical cancer. We found that the effect of SERPINB3KO on both cell death (Fig. 1D-E) and clonogenic survival (Fig. 1F-G) was greater than the radiosensitizing effect seen with cisplatin chemotherapy. Dose modifying factors (DMF, determined as the ratio of the dose resulting in $10 \%$ surviving fraction compared to control) was greater for single gene SERPINB3-KO compared to cisplatin in both SW756 (DMF 0.69 for B3-KO versus 1.01 for cisplatin) and HT3 (DMF 0.73 for B3-KO versus 0.96 for cisplatin), and cisplatin did not further sensitize B3-KO cells to radiation (DMF 0.69 vehicle versus 0.67 cisplatin for HT3, and 0.73 vehicle versus 0.75 cisplatin for SW756).

\section{SERPINB3-KO tumors are more sensitive to radiation in vivo}

To determine the role of SERPINB3 in a p53 mutant cervix tumor in vivo, we established subcutaneous flank xenografts of the HT3 cell line in female athymic nude mice, with either $\mathrm{C}$ or B3-KO clones. Not surprisingly, HT3-C tumors were significantly resistant to radiation, and after a single high-dose fraction of 10Gy we observed no tumor regression, and only one of five HT3 C tumor xenografts with growth delay (Supplemental Fig. 1C). All but 1 of the tumors doubled in size by three weeks after irradiation (Fig. 2A). A dose of $10 \mathrm{~Gy}$ was selected based on in vitro dose response and is within the range of clinically relevant doses for cervix cancer. In contrast, all but one HT3-B3-KO xenografts showed initial tumor regression followed by sustained tumor growth delay (Supplemental Fig. 1D), and less than $50 \%$ of the tumors doubled over the course of the experiment. Additionally, four of five tumors in the irradiated HT3-C group also developed ulcers requiring sacrifice compared to none of the irradiated HT3-B3-KO tumors 
(asterisks). Days to tumor doubling was significantly different for the HT3-B3-KO irradiated tumors but not for the HT3-C tumors (Fig. 2B).

SW756 xenograft tumors were more radiosensitive; a single fraction of 10Gy induced tumor growth delay in both $\mathrm{C}$ and B3-KO tumors (Supplemental Fig. 1E, F). Fewer radiated B3-KO tumors reached preradiation size compared to $\mathrm{C}$ tumors (Fig. $2 \mathrm{C}$ ), and tumor doubling time was significantly longer in the B3$\mathrm{KO}$ irradiated tumors but not the $\mathrm{C}$ irradiated tumors (Fig. 2D). Of note, radiation was administered to B3$\mathrm{KO}$ tumors 10 days later than $\mathrm{C}$ tumors because the B3-KO tumors exhibited slower initial tumor growth rate (Supplemental Fig. 1E, F).

\section{Increased radiation-induced cell death in B3-KO cells is not explained by cell cycle distribution or compromised repair of DNA double strand breaks}

Sensitivity to radiation-induced death can vary depending on the phase of cell cycle at the time of treatment. Therefore, we analyzed cell cycle distribution of $\mathrm{C}$ and B3-KO cells without RT and 48 hours after 4Gy by quantifying DNA content. Although some absolute differences were significant, we found no meaningful differences in cell cycle distribution either de novo or following 4Gy to explain the differential radiosensitivity (Fig. 3A-D). Sub-G1, sham-treated S-phase (HT3 and SW756) and G2/M phase (SW756) populations were statistically different between the $\mathrm{C}$ and B3-KO cells; however, the differences were small in magnitude, and if meaningful, would suggest B3-KO cells should be more sensitive to RT, the opposite of our observations (Fig. 3B, D). For instance, SW756 B3-KO sham-treated cells had roughly 40\% of cells in S-phase, compared to $32 \%$ in C cells; however, cells in S phase are usually more sensitive to the molecular effects of irradiation, thus one would expect the $C$ cells would be more sensitive to radiationinduced death, which is not what we observe. In response to radiation, accumulation of cells in the G2/M phase was not different in C and B3-KO cells with the SW756 cell line background (HPV positive, p53 wild type). Cell cycle distribution did not change significantly following RT for either HT3 C or B3-KO cells (HPV negative, p53 mutant), consistent with aberrant cell cycle checkpoint response commonly seen in p53 mutant cells (Fig. 3A, B). While the subtle differences in baseline cell cycle distribution between B3$\mathrm{KO}$ and $\mathrm{C}$ cells are interesting and a focus of future studies, they do not appear to result in increased radiation sensitivity of $\mathrm{B} 3-\mathrm{KO}$ cells.

Reduced capacity to repair double strand DNA breaks induced by ionizing radiation results in decreased clonogenic survival. Thus, in order to determine if DNA damage repair capacity contributes to radiosensitivity of B3-KO cells, we quantified gamma-H2AX ( ${ }^{\mathrm{H}} \mathrm{H} 2 \mathrm{AX}$ ) foci in $\mathrm{C}$ and B3-KO cells treated with sham or 2Gy RT at 30 minutes and 24 hours post treatment as a surrogate for repair of DNA-damage. As expected, increased $\triangle \mathrm{H} 2 \mathrm{AX}$ foci were observed at the 30 minute time point in all cell lines (foci/nucleus increased 4-5 fold, Fig. 3E). Foci resolved to sham levels by 24 hours in all cell backgrounds, suggesting that the DNA-damage repair machinery is functioning similarly in both $\mathrm{C}$ and B3-KO cells. Interestingly, the mean number of foci per nucleus was moderately but reproducibly higher in HT3-B3-KO cells compared to HT3-C cells at 30 minutes post-2Gy, potentially indicating higher direct DNA damage in these cells, or perhaps more likely, different kinetics of response to DNA-damage (Fig. 3E). Representative 
fluorescent images are shown in Fig. 3F. We also evaluated phosphorylation of ataxia-telangiectasia mutated (ATM) by Western blot as an indicator of response to DNA-damage; levels of phosphorylatedATM were increased 30 minutes after 2 Gy radiation, and decreased by 24 hours in all cell backgrounds (Fig. 3G, H). There was no apparent difference in 30 minute ATM phosphorylation between HT3-C and B3-KO cells.

\section{Cell death in B3-KO cells following RT is primarily necrotic}

While ionizing radiation is known to induce cell death in tumour cells, the precise mechanism of this demise is unclear and could potentially involve multiple cell death pathways. Thus, we took a multifaceted approach to determine which cell death mechanism(s) occur in response to radiation in SERPINB3-C and B3-KO cells over time. First, we evaluated cells with transmission electron microscopy (TEM) to determine if cell death morphology induced by ionizing radiation was primarily apoptotic or necrotic. Untreated C and B3-KO cells showed cells with large nuclear:cytoplasmic ratio as expected, without apparent differences in morphology (Fig. 4A, B, sham). In contrast, when treated with IR, phenotypic differences in $\mathrm{C}$ and B3-KO cells were striking. Dead B3-KO cells displayed necrotic morphology in both HT3 and SW756 (Fig. 4A, B, 10Gy), with swollen and disintegrating nuclei (note size of B3-KO 10Gy nucleus is $~ 20-30 \mu \mathrm{M}$ in diameter compared to $10-15 \mu \mathrm{M}$ in sham-treated and C 10Gy conditions), and highly vacuolated cytoplasm. High magnification images show breaks in the plasma membrane, cytoplasmic clearing, and largely normal mitochondrial morphology (Fig. 4A, B, inset). Irradiated HT3-C and SW756-C cells displayed enlarged cells with more frequent mitochondria, intact nuclear envelop. Blinded scoring of randomly sampled TEM images by two reviewers shows a significantly higher proportion of cells with necrotic morphology in irradiated B3-KO cells ( 25\%) compared to irradiated C cells ( $<5 \%$ ) (Fig. $4 \mathrm{C}$ ). We also noted that the vacuoles seen in the irradiated B3$\mathrm{KO}$ cells were membrane-bound, consistent with lysosomes, and in many cases the membranes were ruptured, releasing their contents into the cytoplasm (Fig. 4D, E).

Second, cells were treated with increasing doses of RT and analyzed by Western blot and fluorescent microscopy at 24 hour intervals up to 96 hours. Western blots were probed for markers of multiple regulated cell death pathways including cleavage of end-effector caspase-3 and caspase-7 (apoptosis), and Poly (ADP-ribose) polymerase (PARP) cleavage products (apoptosis), gasdermin D (GSDMD) cleavage to $\mathrm{p} 30$ pore-forming product (pyroptosis), and phosphorylation of mixed lineage kinase domain like pseudokinase (MLKL) (necroptosis), and receptor interacting serine/threonine kinase 3 (RIPK3) (necroptosis). Of note, there are no accepted markers of ferroptosis that can be determined by Western blot. Cell death was quantified in parallel using Sytox exclusion and positive controls for caspase and

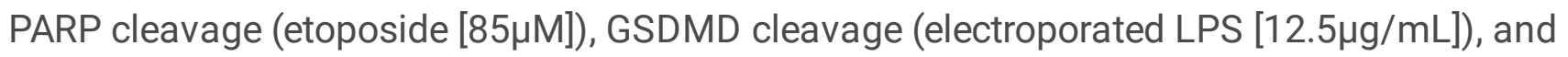
MLKL/RIPK3 phosphorylation (TNFa [20ng/mL] + BV6 [100nM] + ZVAD [50 $\mu \mathrm{M}]$ ) were performed simultaneously. In HT3 cells, caspase-3 (CC-3), caspase-7 (CC-7) and PARP cleavage was detected at later time points with an apparent dose response (Fig. 5A). There was qualitatively more caspase-3/7 cleavage in HT3-B3-KO cells compared to $C$ cells, though low compared to the amount of cell death seen at those time points and compared to the apoptosis positive control (Fig. 5A, Supplemental Fig. 2C). In SW756 cells, no caspase-3 or caspase-7 cleavage was detected even 96 hours after 30Gy (Fig. 5B). We 
found no evidence of GSDMD cleavage, or p-MLKL, p-RIPK3/RIPK1 (markers of pyroptosis and necroptosis, respectively) in either HT3 or SW756 cells (Fig. 5A, B). Additional controls to demonstrate detection of markers by Western blot are shown in Supplemental Fig. 2C-E, including treatment with inducers and inhibitors of apoptosis (Supplemental Fig. 2C), necroptosis (Supplemental Fig. 2D), and pyroptosis (Supplemental Fig. 2E).

As radiation therapy-induced senescence can also contribute to decreased clonogenic survival, we evaluated if markers of senescence were differentially induced by RT in C and B3-KO cells. Sham-treated SW756-B3-KO cells had slightly higher percent positive senescence associated beta-galactosidase (SABgal) cells compared to SW756-C cells ( 15\% versus 10\%) 24 hours after plating (sham, 0h, Fig. 5C, D); however, there was no difference 96 hours after sham-treatment, and SA- $\beta$ gal positive cells were not significantly increased in either SW756-C or-B3-KO cells after 30Gy RT (Fig. 5C, D). HT3 cells did not have any SA- $\beta$ gal positive cells in any fields of view either with sham or 10Gy RT (Supplemental Fig. 3). Similarly, Western blot analysis for BCL-2 and BAX, which are commonly increased and decreased, respectively, in senescent cells, were not significantly different between $\mathrm{C}$ and B3-KO cells, and did not change in either direction following treatment with ionizing radiation (Fig. 5E).

Thirdly, pharmacologic inhibitors of pan-caspase activity (qVD-OPH [1uM]), necroptosis (necrostatin-1 $[10 \mu \mathrm{M}])$, pyroptosis $(\mathrm{VX}-765,50 \mu \mathrm{M})$, and ferroptosis (ferrostatin-1 [50nM]) were incubated with cells beginning 24 hours after treatment with ionizing radiation. The pan-caspase inhibitor qVD-OPH attenuated cell death in both C and B3-KO cells by about $10 \%$ in HT3 cells, but not SW756 cells (Fig. 5F). Necrostatin-1, ferrostatin-1 and VX-765 did not inhibit radiation-induced cell death in either C or B3-KO cells in either cell background (Fig. 5F).

Since caspase-3 and caspase-7 cleavage was detected in vitro, we investigated hallmarks of apoptotic cell death in tumors in vivo following radiation. Flank xenografts were established in nude athymic mice from the HT3 cell lines as described above, randomized to sham or 10Gy radiation, and harvested 96 hours post-RT. Percent TUNEL positive nuclei and IHC for cleaved-caspase 3 (CC3) was quantified on tissue sections. Absolute levels of TUNEL positive nuclei (Fig. 5G, representative images Fig. 5H-K) and CC3 positive cells (Fig. 5L, representative images Fig. 4M-P) were low $(<\sim 10 \%)$ and were not significantly different between $\mathrm{C}$ and B3-KO cells.

\section{Radiation induces loss of lysosomal membrane integrity that precedes cell death in SERPINB3-deficient cells}

C. elegans genetically lacking srp-6 (homologue of human SERPINB3) demonstrate extensive lysosomal membrane permeability (LMP) in their intestinal epithelial cells leading to intestinal cell death and ultimately organismal death when exposed to various cytotoxic stressors ${ }^{17}$. This cell death mechanism has been further characterized and termed lysoptosis (citation for Good et al, Comms Biology). Given the conserved molecular function of SERPINB3 as an intracellular lysosomal cysteine protease inhibitor, we hypothesized that cervical tumor cells lacking SERPINB3 would be susceptible to LMP and lysosomal rupture following an insult such as ionizing radiation. TEM of irradiated B3-KO cells showed evidence of 
vesicular membrane rupture reminiscent of LMP in both HT3 and SW756 cells (Fig. 4D, E). To determine if lysosomal membrane integrity was lost prior to cell death or occurred as a post-mortem event, we performed live-cell time-lapse confocal microscopy to observe cells in the process of dying. Lysosomes were marked with the acidophilic dye LysoTracker ${ }^{\text {TM}}$-deep red (green), and cell membrane permeability as a measure of cell death was determined by propidium iodide (red). C or B3-KO cells were treated with $10 \mathrm{~Gy}$ and imaged during a period of expected high percent death, beginning approximately 72 or 96 hours after treatment. Dying B3-KO cells treated with radiation lost lysosomal integrity prior to loss of cell membrane integrity (Fig. 6A, C). HT3-C cells, in contrast, showed a delayed loss of Lysotracker staining after becoming PI positive (Fig. 6B, D).

Treatment of cells with the cysteine protease inhibitor E64d inhibited radiation-induced cell death in HT3$\mathrm{C}$ and to a greater degree in HT3-B3-KO cells (Fig. 6E), providing further supporting evidence that lysosomal protease activity is required for cell death, particularly in the absence of SERPINB3.

\section{The reactive site loop of SERPINB3 is required to protect cervical cancer cells against radiation-induced death and to promote tumor growth}

To determine if SERPINB3 is sufficient to increase radioresistance in cervical cancer cells, SiHa (HPV16+/p53 wild type) and C33A (HPV-/p53 mutant) cell lines with no detectable SERPINB3 protein were used to generate stable clones expressing the wild-type SERPINB3 (B3) or an empty vector control (VC), with bicistronic expression of green fluorescent protein (GFP) (Fig. 7A). Clonogenic survival was significantly higher in SiHa-B3 cells compared to SiHa-VC cells (DMF 1.25, Fig. 7B). C33A cells grow in a manner that is partially adherent with a population of viable cells that easily detach from the tissue culture dishes used for clonogenic survival assays, leading to high variability in the assay. Use of soft agar suspension resulted in disaggregated colonies with similar challenges. Therefore, the CellTiter-Glo® ATP-dependent reagent was employed to estimate cell viability in C33A cells. Indeed, we find that C33AB3 cells have higher cell viability following radiation treatment compared to C33A-VC cells, with similar findings in the SiHa background (Fig. 7C).

To determine if the protease-inhibitory function of SERPINB3 is required for radioprotection in these cells, we generated isogenic stable clones expressing SERPINB3 with a single alanine to arginine amino acid substitution at the $\mathrm{P} 14$ residue (Schechter and Berger numbering scheme ${ }^{23}$ ) of the C-terminal reactive site loop (RSL), corresponding to amino acid 341 in the hinge region (termed B3-A341R). The resultant protein blocks loop insertion after cleavage by target proteases and thus does not inhibit protease activity ${ }^{7}$. For functional analyses, a clone with similar levels of B3-A341R protein expression compared to the B3expressing clone was selected (Fig. 7A), and clonogenic survival and cell viability was compared to B3and VC-containing cell lines (Fig. 7B, C). Expression of B3-A341R did not protect cells from radiation and survival was similar to VC-expressing cells (DMF 0.93 compared to VC). Flank xenograft tumors of the C33A cell lines were established to determine effect of B3 and B3-A341R on tumor growth and in vivo radiation resistance. Sham treated C33A-B3 tumors overall grew faster than VC or B3-A341R tumors (Fig. 7D). After tumor establishment, half of the mice were randomized to tumor-directed radiation, and 
B3 tumors continued to grow after a single dose of 10Gy compared to VC and B3-A341R tumors (Fig. 7E). Tumor doubling time was not significantly different in sham-treated versus 10Gy irradiated B3-expressing tumors, whereas time to tumor doubling was significantly longer in irradiated VC- and A341R-containing tumors (Fig. 7F). Images of dissected tumors to portray relative tumor size at the time of sacrifice for sham irradiated and 10Gy treated tumors are shown in Fig. 7G. Taken together, these data suggest that SERPINB3 protects cells from ionizing radiation by inhibiting target lysosomal cysteine proteases.

\section{Discussion}

Since its isolation from human cervical tumors, SCCA has been shown to serve as a strong and consistent indicator of poor outcomes in cervical cancer and other types of cancers. While others have shown that SCCA1/SERPINB3 promotes tumor growth and resistance to cytotoxic agents, these data are the first to show that SERPINB3 is directly responsible for radiation resistance of cervical cancer cells by inhibiting a lysosome mediated necrotic cell death pathway. Moreover, we show that the effect of SERPINB3 loss on radiation sensitivity in cervical tumor cells is similar if not greater than cisplatin, currently used as the standard of care to radiosensitize cervical cancer. These findings suggest that SERPINB3 may serve as a therapeutic target for radiosensitization of resistant cervical cancers. Additionally, the data also implicate a lysosome-dependent regulated cell death pathway in radiationinduced cell death and resistance.

lonizing radiation has a host of direct and indirect cellular effects on tumor cells ultimately leading to recovery and survival, or cell death. Direct damage to DNA and other macromolecules is known to lead to the induction of apoptosis primarily in hematologic cells, but apoptosis is not a predominant mode of cell death in most solid tumors. Following IR, the inability of the cell to undergo mitosis secondary to dysfunctional cell cycle checkpoints and accumulated unrepaired DNA-damage, entering a state known as mitotic catastrophe, thought to ultimately lead to cellular demise by secondary death pathways, or to enter a senescent state ${ }^{24}$. However, the dynamics of radiation-induced cell death, and the exact molecular mechanisms contributing to the cell fate decision are complex. The most recent recommendations from the Nomenclature Committee on Cell death describes myriad cell death subroutines, including cellintrinsic and -extrinsic modes ${ }^{25}$. Importantly, the committee stresses that there is often a great deal of interconnectivity between signal transduction cascades leading to one cell death mode versus another. Therefore, in order to truly understand the relative contributions of various lethal regulated cell death programs to radiation-induced tumor cell kill, it is critical to evaluate not only the presence of individual markers of cell death pathways at single time points and under single conditions, but multiple markers of suspected death modes at varying time points and dose levels. As such, in this study we undertook a broad evaluation to determine the intrinsic cell death routines contributing to radiation-induced death in SERPINB3-KO cells compared to control cells. The goal was to identify vulnerabilities exposed by the loss of SERPINB3 that can be exploited for therapeutic radiosensitization.

The first finding was the prevalence of necrotic morphology in SERPINB3-KO cells following ionizing radiation. Although some end-effector caspase cleavage was detected by Western blot analysis, the 
amount was qualitatively low compared to the percent cell death observed. Pyroptosis is a form of necrotic regulated cell death typically induced by microbial pathogens, whereby cleavage of gasdermin $D$ (GSDMD) by caspase-1, or in some cases gasdermin E (GSDME) cleavage by caspase-3, mediates its localization to the cell membrane. GSDMD-N or GSDME-N oligomerization forms pores resulting in rapid permeabilization of the cell membrane ${ }^{26}$. Despite some caspase-3 cleavage, we found no evidence of GSDMD or GSDME cleavage following radiation in either B3-C or B3-KO cells. Ferroptosis is another necrotic RCD which occurs independently of caspase activation and relies heavily on generation of ROS, a hallmark of ionizing radiation ${ }^{27}$. Nevertheless, ferrostatin-1 did not inhibit radiation-induced cell death in either $\mathrm{C}$ or B3-KO cells, and TEM images of necrotic cells revealed largely intact mitochondria suggesting ferroptosis is not a predominant form of radiation-induced cell death in B3-KO cells.

In C. elegans, the orthologue of SERPINB3, srp-6, serves as an intracellular cysteine protease inhibitor and was previously shown to protect animals against diverse toxic stressors by inhibiting lysosomal proteases and subsequent animal death ${ }^{17}$. In addition to hypo-osmotic conditions and heat shock, we also demonstrated that srp-6 null animals were more sensitive to oxidative stress and hypoxia. Additionally, several groups have shown that DNA-damage induced by topoisomerase inhibitors camptothecin and etoposide causes lysosomal membrane permeability ${ }^{28,29}$. Therefore, we hypothesized that radiation, which causes both DNA damage and accumulation of oxidative species, might induce LMP in cervical cancer cells, particularly in cells lacking SERPINB3. Indeed, we observed TEM evidence of lysosome-like vesicle membrane rupture in B3-KO tumor cells treated with ionizing radiation, and loss of lysosomotropic staining by live cell microscopy in dying cells, similar to that seen in srp-6 null $C$. elegans. Further supporting the idea that SERPINB3 lysosomal protease inhibitor activity is responsible for its ability to protect cells against radiation, we found that the pharmacologic cysteine protease inhibitor E64d abrogated cell death in B3-KO cells. Additionally, expression of wild type but not the RSL mutant B3A341R protected SERPINB3-low cervical cancer cells from radiation further supporting the importance of an intact RSL to bait-and-trap target lysosomal proteases released from the lysosome after exposure to ionizing radiation.

While the DNA-damage repair capacity appears intact in B3-KO cells, suggesting that inability to repair DNA-damage is not contributing to the radiosensitivity observed in these cells, we did observe that the initial quantity of $\triangle \mathrm{H} 2 \mathrm{AX}$ foci, which is a surrogate for number of double strand DNA breaks, was higher in B3-KO cells specifically in the HT3 background. The mechanism of this is not clear and is under current scrutiny; however, the myeloid and erythroid nuclear termination protein (MENT), a chicken Clade B or intreacellular SERPIN, is associated with heterchromatin ${ }^{30}$, thus there is precedence for nuclear function of SERPIN intracellular family proteins. Moreover, cysteine cathepsin proteases have been reported to localize to the nucleus ${ }^{31,32}$. It is conceivable that nuclear cathepsins may have a role in vulnerability to DNA-damage that is modifiable by the presence or absence of SERPINB3. Perhaps more likely is a slightly different kinetics of foci formation in the $\mathrm{C}$ and $\mathrm{B} 3-\mathrm{KO}$ cells that will be discerned in future detailed analysis. 
Importantly, we found similar effects of SERPINB3 on radiation-induced cell death and resistance in cervical tumor cell lines that are HPV positive and negative, and p53 wild type and mutant. These findings suggest that radiation-induced lysosome mediated necrosis proceeds in a manner that is not dependent on functional p53.

The major limitation of the current study is the experimental difficulty posed by the long time course of radiation-induced death. Instead of minutes to hours required for classic cell death inducers to result in cellular demise, most non-hematologic cells treated with ionizing radiation do not die for at least 48 hours after treatment, and in many cases several days after a single treatment. While we made every effort to capture an accurate view of cell death modes during each phase of this long time course, and in response to various doses of radiation, it is possible that less prevalent cell death events were not captured by Western blot, live cell imaging and TEM assays. Additionally, the time required to observe a single cell death event using live cell imaging inherently introduced difficulty in tracking cells while maintaining confocal resolution. Finally, the complexity of signal transduction occurring in the hours to days after treatment with ionizing radiation and the long time course of the experiments likely complicates the interpretation of pharmacologic inhibitors of cell death.

While we are currently investigating the possibility, it remains unclear if other cancer subtypes expressing SERPINB3 are protected against radiation in a similar manner. Given the available evidence that SERPINB3 expression in many other cancers including epithelial cancers of the head and neck, anus, lung, esophagus as well as hepatocellular carcinoma carries a similar poor prognosis, we expect this to be the case.

Taken together, this body of evidence contributes to the understanding of regulated cell death occurring in tumor cells in response to therapeutic ionizing radiation, and identified a targetable vulnerability, SERPINB3.

\section{Methods}

\section{Cell lines and tissue culture}

All cell lines were obtained from ATCC and grown in monolayer at $37^{\circ} \mathrm{C} / 5 \% \mathrm{CO} 2$ in Dulbecco's modified Eagle's medium (DMEM) or Iscove's Modified Dulbecco's Medium (IMDM) supplemented with $10 \%$ fetal bovine serum, $0.1 \mathrm{mg} / \mathrm{mL}$ penicillin, 100 units $/ \mathrm{mL}$ streptomycin, and $15 \mathrm{mM}$ Hepes. Generation and characterization of B3-KO and isogenic CRISPR-control cell lines SW756 and HT3 was described previously ${ }^{11}$. In brief, a single-vector lentiviral system was used, driving expression of Cas 9 and the gRNA sequence. KO was confirmed by sequencing of the SERPINB3 gene, Western blot showing no protein product, and sequencing of most-likely off-target genes to confirm specificity. For the current paper, $\mathrm{SiHa}$ and C33A cell lines were engineered to stably express pULTRA (Addgene 24129) mammalian vector driving expression of the wild type SERPINB3 gene, or SERPINB3 mutant encoding a single alanine to arginine substitution at amino acid 341 (B3-P14m). 


\section{Irradiation of cell lines and mice}

Cells in dishes were irradiated at a dose-rate of 300cGy per minute using the RS-2000 Biological System (Rad Source, Suwanee, GA), calibrated once monthly by a medical physicist. Sham irradiation conditions were transported to the irradiator and placed at room temperature conditions while delivering radiation. Mouse xenograft tumors were irradiated using the Xstrahl Small Animal Radiation Research Platform (SARRP) 200 (Xstrahl Life Sciences, Suwannee, GA). After being fitted with a nose cone, mice were individually subjected to isoflurane anesthesia and imaged by on-board micro-computed tomography (CT). CT images imported into Muriplan were used to select an isocenter. The tumor was then irradiated using anterior-posterior opposed beams using the $10 \mathrm{~mm} \times 10 \mathrm{~mm}$ collimator at a dose rate of $3.9 \mathrm{~Gy} / \mathrm{min}$. Sham irradiated mice were transported to the SARPP facility and remained in the room at the same conditions during radiation of the irradiated mice.

\section{Cell cycle analysis}

Cells were seeded at $0.15 \times 10^{\wedge} 6$ cells per well and treated 24 hours later with $4 \mathrm{~Gy}$ or Sham RT. Cells were fixed and permeabilized with Triton X-100 48 hours later (after optimization of IR dose and incubation time), stained with propidium iodide, and cell cycle distribution determined using flow cytometry of DNA content. FlowJo ${ }^{\text {TM }}$ Software (Becton, Dickinson and Company, Ashland, OR, USA) was used to analyze and visualize the data.

\section{Gamma-H2AX foci formation assay}

Cells were seeded in 4-well chamber slides (Nunc® Lab-Tek ${ }^{\circledR}$ II Chamber Slide ${ }^{\mathrm{TM}}$, C6807, Millipore Sigma, St Louis, MO, USA) to 50-60\% confluence and allowed to adhere to the slide for 24 hours. Cells were then treated with sham or 2Gy radiation as described in the "Irradiation of cell lines and mice" section and incubated at $37^{\circ} \mathrm{C}$ for 30 minutes or 24 hours prior to washing with PBS and fixation with $3 \%$ PFA. Cells were then permeabilized with $0.1 \%$ Triton-X and washed with PBS before blocking with blocking buffer (10\% FBS, $0.5 \%$ BSA in PBS) for one hour at room temperature. Slides were incubated at $4^{\circ} \mathrm{C}$ overnight with 1:300 anti-gH2AX antibody (05-636, Millipore Sigma, St Louis, MO, USA). Slides were washed in three changes of PBS and incubated with 1:500 anti-mouse-lgG AlexaFluor 488 conjugated secondary antibody for 3 hours at room temperature. Slides were mounted with VECTASHIELD ${ }^{\circledR}$ HardSet $^{\text {TM }}$ Antifade Mounting Medium with DAPI ( $\mathrm{H}-1500$, Vector Laboratories, Burlingame, CA, USA). Fluorescent images were obtained on the Zeiss LSM510. Images were then analyzed using ImageJ Software to quantitate foci per nucleus for a random selection of six 100X fields of view, corresponding to $30-50$ cells per condition.

\section{Cell death assay}

Cells were seeded in order to achieve $50-70 \%$ confluence 24 hours later in a 96-well glass-bottom, opaque-walled dish (Greiner Bio-One ${ }^{T M}$ CellStar $^{T M} \mu$ Clear $^{\text {TM }}$ 96-Well, Cell Culture-Treated, Flat-Bottom Microplate, Fisher, 7000166). 24 hours after plating, plates were treated with varying doses of IR or Sham, 
with one-hour pre-incubation of inhibitors where indicated. Cisplatin was added 1 hour prior to radiation, where indicated, at the half-maximal inhibitory concentration (IC50) of cisplatin monotherapy determined by Alamar blue assay $\left(0.5 \mu \mathrm{M}\right.$ for SW756 and $0.01 \mu \mathrm{M}$ for HT3). At established time points, Sytox ${ }^{\mathrm{TM}}$ orange (1:30,000, Fisher Scientific, S11368) and Hoescht 33342 (1:2,000) were added 15-30 minutes prior to quantitative imaging on the Cytation ${ }^{\text {TM }} 5$ multi-mode reader (BioTek® Instruments, Inc., Winooski, Vermont). Percent dead cells was determined by optimized automated counting of Sytox-positive cells divided by Hoescht-positive nuclei, averaged over triplicate wells.

\section{Western blot}

Cells were seeded and treated as in the cell death assay methods, except scaled to 6-well or $10 \mathrm{~cm}$ dishes in order to obtain adequate cell lysate. At established time points, coinciding with the cell death assay, cells were lysed with Cell Lysis Buffer (Cell Signaling Technology, Danvers, MA) supplemented with proteinase/phosphatase inhibitors and PMSF. Equal parts protein (25-30 $\mu$ g) and Laemmli sample buffer (Santa Cruz Biotechnology, Dallas TX) were boiled at 95 degrees Celsius for 10 minutes and gel electrophoresed on 4-20\% gradient gels (Mini-Protean TGX, Bio-Rad, Hercules, CA), transferred to PVDF blot using the Trans-Blot Turbo Transfer system (Bio-Rad, Hercules, CA), blocked with 5\% milk:TBS-Tween and incubated with 1:4,000 anti-SCCA1 antibody (NBP2, Novus International, Saint Louis, MO) overnight at four degrees Celsius, 1:100,000 anti-Actin (A5441, Santa Cruz Biotechnology, Dallas, TX), 1:2,000 antiGAPDH-HRP conjugated antibody (D16H11, Cell Signaling Technology, Danvers, MA) for 2 hours at room temperature, 1:1000 anti-GSMDC1 (NBP2-33422, Novus Biologicals, LLC, Littleton, CO) overnight at four degrees Celsius, 1:1000 anti-p-MLKL (91689S, Cell Signaling Technology, Danvers, MA) overnight at four degrees Celsius, 1:1000 anti-Cleaved Caspase3 (9661, Cell Signaling Technology, Danvers, MA) overnight at four degrees Celsius, 1:1000 anti-Cleaved Caspase7 (8438, Cell Signaling Technology, Danvers, MA) overnight at four degrees Celsius, 1:1000 anti-Cleaved PARP (5625s, Cell Signaling Technology, Danvers, MA) overnight at four degrees Celsius, 1:1000 anti-Full Length PARP (9524s, Cell Signaling Technology, Danvers, MA) overnight at four degrees Celsius, 1:1000 anti-Caspase7 (12827, Cell Signaling Technology, Danvers, MA) overnight at four degrees Celsius, 1:500 anti-Caspase3 (sc-7272, Santa Cruz biotechnology, INC, Texas) overnight at four degrees Celsius, 1:1000 anti-RIP3 (57220, Cell Signaling Technology, Danvers, MA) overnight at four degrees Celsius, 1:1000 anti-RIPK3 (ser227) (93654s, Cell Signaling Technology, Danvers, MA) overnight at four degrees Celsius, 1:1000 anti-RIPK1 (ser166) (44590, Cell Signaling Technology, Danvers, MA) overnight at four degrees Celsius, 1:10000 anti-p-ATM (ab81292100UL, Abcam, Cambridge, MA) overnight at four degrees Celsius, 1:1000 anti-ATM (NB100-309, Novus Biologicals, LLC, Littleton, CO) overnight at four degrees Celsius. Anti-mouse or anti-rabbit HRPconjugated secondary antibody was used for detection with ECL chemiluminescent reagent (GE Healthcare Life Sciences, Pittsburgh, PA), visualized, and quantified using the Bio-Rad ChemiDoc MP imaging system and Image Lab software (Bio-Rad, Hercules, CA).

\section{Clonogenic cell survival assay}


500-1000 cells per well were seeded in 6-well plates 24 hours prior to treatment with increasing doses of radiation $(2,4,6$ Gy $\times 1)$ and incubated for $1-3$ weeks until control plates formed visible colonies $(\geq 50$ cells). IC50 concentration of cisplatin was added 1 hour prior to radiation, where indicated. Plates were fixed and stained with $0.5 \%$ Crystal Violet, 30\% Methanol, 10\% Acetic Acid, $60 \%$ ddH2O for 30 minutes, rinsed in tap water and air dried at room temperature. Surviving fraction was calculated as the number of colonies $\div$ (500 * plating efficiency) and plotted on a $\log _{10}$ scale as per convention. The linear quadratic equation was fit to each dataset using GraphPad Prism 8๑. The dose modifying factor (DMF) was determined as the ratio of the dose resulting in $10 \%$ surviving fraction compared to control, indicated as the reference condition.

\section{Cell Titer-Glo survival assay}

Cells were seeded to $50-70 \%$ confluence in 96 -well dishes and cultured overnight prior to treatment with increasing doses of ionizing radiation. 24 hours later cells were washed once with PBS on ice then lysed directly in the well using Cell Titer-Glo reagent and incubated per manufacturer's protocol prior to measurement of luminescence on the SpectraMax i3 plate reader. Mean relative light units (RLUs) normalized to background value of a well with no cells was plotted with standard deviation.

\section{Time lapse confocal microscopy}

Cells were seeded as described in the cell death assay except on Nunc ${ }^{T M}$ Lab-Tek $^{\text {TM }}$ II 8-well Chambered Coverglass (155409PK, Thermo Scientific ${ }^{\mathrm{TM}}$ ) or $35 \mathrm{~mm}$ cover glass culture dishes (MatTek Life Sciences, P35G-1.5-14-C), irradiated approximately 24 hours after seeding and placed on the Leica TCS SP8 X confocal microscope (Leica Microsystems Inc., Buffalo Grove, IL) beginning 24, 48, 72 or 96 hours after IR and imaged every 20-30 minutes using a 63x1.4N.A. oil objective for approximately 16 hours at a time at $37^{\circ} \mathrm{C}$ in $5 \% \mathrm{CO} 2$ using an OKO-labs cage incubator. Prior to imaging, cells were stained with LysoTracker $^{T M}$ Deep Red (Invitrogen ${ }^{T M}$, Life Technologies Corp., Carlsbad, CA) for 15-30 minutes, washed twice with PBS and stained with propidium iodide (1:500) in FluoroBrite DMEM media (Invitrogen, Life Technologies Corp., Carlsbad, CA) with 10\% FBS and 4mM L-glutamine. Images were collected using LASX software (Leica Microsystems, Buffalo Grove, IL) and visualized and analyzed using Volocity software (v6.3.5, Quorum). Image contrast and brightness were adjusted for presented images in Microsoft PowerPoint with similar adjustments for all images in the experiment (acquired with identical microscope settings). As acquired images available for review.

\section{Transmission electron microscopy}

Cells were plated in $60 \mathrm{~mm}$ dishes to reach a confluence of $\sim 70 \%$ at the time of RT, then treated 24 hours after plating with sham, 10Gy (HT3) or 30Gy (SW756) RT. Cells were harvested by trypsinization 96 hours after treatment. For ultrastructural analyses, samples were fixed in $2 \%$ paraformaldehyde/ $2.5 \%$ glutaraldehyde (Polysciences Inc., Warrington, PA) in 100 mM sodium cacodylate buffer, $\mathrm{pH} 7.2$ for 1 hour at room temperature. Samples were washed in sodium cacodylate buffer at room temperature and postfixed in 1\% osmium tetroxide (Polysciences Inc.) for 1 hour. Samples were then rinsed extensively in 
$\mathrm{dH}_{2} \mathrm{O}$ prior to en bloc staining with $1 \%$ aqueous uranyl acetate (Ted Pella Inc., Redding, $\mathrm{CA}$ ) for 1 hour. Following several rinses in $\mathrm{dH}_{2} \mathrm{O}$, samples were dehydrated in a graded series of ethanol and embedded in Eponate 12 resin (Ted Pella Inc.). Sections of $95 \mathrm{~nm}$ were cut with a Leica Ultracut UCT ultramicrotome (Leica Microsystems Inc., Bannockburn, IL, USA), stained with uranyl acetate and lead citrate, and viewed on a JEOL 1200 EX transmission electron microscope (JEOL USA Inc., Peabody, MA, USA) equipped with an AMT 8 megapixel digital camera and AMT Image Capture Engine V602 software (Advanced Microscopy Techniques, Woburn, MA).

\section{In vivo tumor growth and radiation response}

Female athymic nude mice were innoculated at $7-10$ weeks of age with $0.5 \times 10^{6}$ cells suspended in a 1:1 mixture of IMDM media and Matrigel $\circledast$ Matrix (Corning, Glendale, AZ, USA) subcutaneously in the flank region. Tumor growth was measured by caliper twice weekly beginning at 3 days post-innoculation to determine estimated tumor volume assuming an ellipsoid sphere $\left(V=0.5^{\star}\left(L \times W^{\wedge} 2\right)\right)$, where $V=$ volume, $L$ = length of the long-axis, $\mathrm{W}=$ width of the short axis. Once tumors reached an appropriate size, animals were randomized to treatment with indicated doses of IR or sham IR on the SARRP as described in "Irradiation of cell lines and mice" section. Following monitored recovery from anesthesia, tumor growth was measured by caliper twice weekly until tumors reached a maximal dimension of $20 \mathrm{~mm}$ or otherwise reached a pre-defined sacking criterion of skin ulcer.

\section{Tumor tissue analysis by histology and immunohistochemistry}

At tumor harvest, mice were sacrificed using a $\mathrm{CO}_{2}$ asphyxiation chamber and tumors immediately harvested and divided into three components for flash freezing, into $4 \%$ paraformaldehyde (PFA), and fixation buffer for TEM as described above. PFA-fixed tumor was sent and histology was performed by HistoWiz Inc. (histowiz.com) using a Standard Operating Procedure and fully automated workflow. Samples were processed, embedded in paraffin, and sectioned at $4 \mu \mathrm{m}$. Immunohistochemistry was performed on a Bond Rx autostainer (Leica Biosystems) using standard protocols. Antigen retrieval method was heat induced epitope retrieval (HIER) at $\mathrm{pH}=6.0$ for 20 minutes (CC3) and enzyme digestion for 10 minutes (TUNEL). Antibodies used were anti-cleaved caspase-3 (Asp175) (Cell Signaling, 9661S, 1:300) and rabbit anti-rat secondary (Vector, 1:100). Bond Polymer Refine Detection Kit (Leica Biosystems, DS9800) was used according to manufacturer's protocol. After staining, sections were dehydrated and film coverslipped using a TissueTek-Prisma and Coverslipper (Sakura). Whole slide scanning (40x) was performed on an Aperio AT2 (Leica Biosystems). TUNEL staining was performed to determine TUNEL positive nuclei as a measure of fragmented nuclear DNA under standard conditions using the Promega DeadEnd Fluorometric Detection System (Promega, G3250). QuPath V0.1.2 open source software was used for digital image viewing and automated analysis using macros for nuclear staining (TUNEL) and cytoplasmic staining (CC3) to determine \% cells positive. Positive control slides processed in batch under identical conditions were used to optimize macros. Full digital slide images are available at https://app.histowiz.com/shared_orders/2ccb0ce8-e4e1-41d1-8f75-a0e621ced36e/slides/ . 


\section{Statistics and reproducibility}

For animal experiments, based on our published and preliminary in vitro data, using a Bonferroni correction with 0.05 significance, $80 \%$ power and a two-sided t-test, we estimated needing 4 mice per group to determine an effect size of 3.5 for tumor growth kinetics. Given an anticipated tumor-take rate of $80 \%$ for HT3 and SW756 cell lines, we injected 5 mice per group for those experiments. Because all of the mice injected developed tumors, we included all in the experiment and analysis. For C33A cell line, tumor take-rate was closer to $100 \%$ so 4 mice per group were injected. Only three mice in the C33A group developed tumors. Tumor volume was estimated based on the two-dimensional caliper measurements and equation for the volume of an ellipsoid sphere: $V=0.5 *\left(L \times W^{2}\right)$, and means were plotted with standard error for the group. Two-way ANOVA with Bonferroni correction was performed to determine difference between mean tumor growth curves. Students' t-test was used to compare groups in flow cytometry, cell death, TEM quantification and histology (CC3 and TUNEL) assays. Individual clonogenic survival data points were fit with the linear quadratic equation, and dose modifying factors (DMF) were calculated at $10 \%$ surviving fraction. In all cases, p-value of $<0.05$ was considered significant.

\section{Declarations}

\section{Acknowledgements}

The authors would like to acknowledge Wandy Beatty in the Molecular Microbiology Imaging Facility, for TEM processing and expert assistance in image acquisition and analysis. TEM experiments were performed in part through the use of Washington University Center for Cellular Imaging (WUCCI) supported by Washington University School of Medicine, The Children's Discovery Institute of Washington University and St. Louis Children's Hospital (CDI-CORE-2015-505 and CDI-CORE-2019-813) and the Foundation for Barnes-Jewish Hospital (3770 and 4642). The authors would also like to thank Dr. Cedric Mpoy and Dr. Buck Rogers of the Small Animal Radiation Research Platform Core, as well as Michael Zahner for his assistance with animal experiments, Dr. Xiaowei Wang for his design of the WU-CRIPSR gRNA Designer which was used for design of the SERPINB3-gRNA, and Lena Zein for administrative assistance. We would also like to thank members of the Washington University Histology Core in the Developmental Biology Department for processing of mouse tumor tissue.

\section{References}

1. Bray, F. et al. Global cancer statistics 2018: GLOBOCAN estimates of incidence and mortality worldwide for 36 cancers in 185 countries. CA. Cancer J. Clin. 68, 394-424 (2018).

2. Siegel, R. L., Miller, K. D. \& Jemal, A. Cancer statistics, 2020. CA. Cancer J. Clin. 70, 7-30 (2020).

3. Kidd, E. A. et al. Clinical outcomes of definitive intensity-modulated radiation therapy with fluorodeoxyglucose-positron emission tomography simulation in patients with locally advanced cervical cancer. Int. J. Radiat. Oncol. Biol. Phys. 77, 1085-1091 (2010). 
4. Tan Mbbs Mrcp Frcr Md, L. T. et al. Image-guided Adaptive Radiotherapy in Cervical Cancer. Semin. Radiat. Oncol. 29, 284-298 (2019).

5. Olsen, J. R. et al. Prognostic utility of squamous cell carcinoma antigen in carcinoma of the cervix: association with pre- and posttreatment FDG-PET. Int. J. Radiat. Oncol. Biol. Phys. 81, 772-777 (2011).

6. Markovina, S. et al. Squamous Cell Carcinoma Antigen Mediates Radiation Resistance in Cervical Cancer. Int. J. Radiat. Oncol. • Biol. • Phys. 99, S146-S147 (2017).

7. Schick, C. et al. The reactive site loop of the serpin SCCA1 is essential for cysteine proteinase inhibition. Proc. Natl. Acad. Sci. U. S. A. 95, 13465-13470 (1998).

8. Olson, S. T. \& Gettins, P. G. W. Regulation of proteases by protein inhibitors of the serpin superfamily. Prog. Mol. Biol. Transl. Sci. 99, 185-240 (2011).

9. Luke, C. et al. Simple modifications of the serpin reactive site loop convert SCCA2 into a cysteine proteinase inhibitor: a critical role for the P3' proline in facilitating RSL cleavage. Biochemistry 39, 7081-7091 (2000).

10. Koning, P. J. A. de et al. Intracellular Serine Protease Inhibitor SERPINB4 Inhibits Granzyme MInduced Cell Death. PLOS ONE 6, e22645 (2011).

11. Markovina, S. et al. Serum squamous cell carcinoma antigen as an early indicator of response during therapy of cervical cancer. Br. J. Cancer 118, 72-78 (2018).

12. Nakamura, K. et al. The predictive value of measurement of SUVmax and SCC-antigen in patients with pretreatment of primary squamous cell carcinoma of cervix. Gynecol. Oncol. 119, 81-86 (2010).

13. Hsieh, C. Y. et al. Serum squamous cell carcinoma antigen in gynecologic malignancies with special reference to cervical cancer. Taiwan Yi Xue Hui Za Zhi 88, 797-800 (1989).

14. Bolli, J. A. et al. Squamous cell carcinoma antigen: clinical utility in squamous cell carcinoma of the uterine cervix. Gynecol. Oncol. 55, 169-173 (1994).

15. Petrelli, N. J. et al. Squamous cell carcinoma antigen as a marker for squamous cell carcinoma of the anal canal. J. Clin. Oncol. 6, 782-785 (1988).

16. Fassan, M. et al. Squamous cell carcinoma antigen (SCCA) is up-regulated during Barrett's carcinogenesis and predicts esophageal adenocarcinoma resistance to neoadjuvant chemotherapy. Oncotarget 8, 24372-24379 (2017).

17. Luke, C. J. et al. An intracellular serpin regulates necrosis by inhibiting the induction and sequelae of lysosomal injury. Cell 130, 1108-1119 (2007).

18. Murakami, A. et al. Squamous cell carcinoma antigen suppresses radiation-induced cell death. Br. J. Cancer 84, 851-858 (2001).

19. Vakifahmetoglu, H., Olsson, M. \& Zhivotovsky, B. Death through a tragedy: mitotic catastrophe. Cell Death Differ. 15, 1153-1162 (2008).

20. Harima, Y., Sawada, S., Nagata, K., Sougawa, M. \& Ohnishi, T. Human papilloma virus (HPV) DNA associated with prognosis of cervical cancer after radiotherapy. Int. J. Radiat. Oncol. 52, 1345-1351 
(2002).

21. Bossi, P. et al. Treatment-related outcome of oropharyngeal cancer patients differentiated by HPV dictated risk profile: a tertiary cancer centre series analysis. Ann. Oncol. 25, 694-699 (2014).

22. Ang, K. K. \& Sturgis, E. M. Human papillomavirus as a marker of the natural history and response to therapy of head and neck squamous cell carcinoma. Semin. Radiat. Oncol. 22, 128-142 (2012).

23. Schechter, I. \& Berger, A. On the size of the active site in proteases. I. Papain. Biochem. Biophys. Res. Commun. 27, 157-162 (1967).

24. Castedo, M. et al. Cell death by mitotic catastrophe: a molecular definition. Oncogene 23, 2825-2837 (2004).

25. Galluzzi, L. et al. Molecular mechanisms of cell death: recommendations of the Nomenclature Committee on Cell Death 2018. Cell Death Differ. 25, 486-541 (2018).

26. Ding, J. et al. Pore-forming activity and structural autoinhibition of the gasdermin family. Nature 535, 111-116 (2016).

27. Stockwell, B. R. et al. Ferroptosis: A Regulated Cell Death Nexus Linking Metabolism, Redox Biology, and Disease. Cell 171, 273-285 (2017).

28. Emert-Sedlak, L. et al. Involvement of cathepsin D in chemotherapy-induced cytochrome c release, caspase activation, and cell death. Mol. Cancer Ther. 4, 733-742 (2005).

29. Paquet, C., Sané, A.-T., Beauchemin, M. \& Bertrand, R. Caspase- and mitochondrial dysfunctiondependent mechanisms of lysosomal leakage and cathepsin B activation in DNA damage-induced apoptosis. Leukemia 19, 784-791 (2005).

30. Grigoryev, S. \& McGowan, S. Isolation and characterization of the nuclear serpin MENT. Methods Enzymol. 501, 29-47 (2011).

31. Burton, L. J., Henderson, V., Liburd, L. \& Odero-Marah, V. A. Snail transcription factor NLS and importin $\beta 1$ regulate the subcellular localization of Cathepsin $L$ and Cux1. Biochem. Biophys. Res. Commun. 491, 59-64 (2017).

32. Gui, Z. Z. et al. Functional role of aspartic proteinase cathepsin D in insect metamorphosis. BMC Dev. Biol. 6, 49 (2006).

\section{Figures}


A
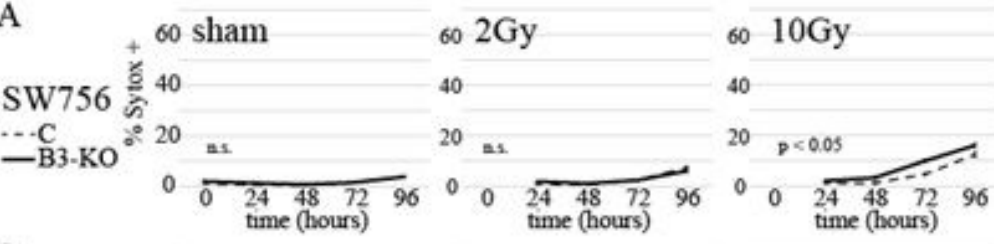

60 30Gy
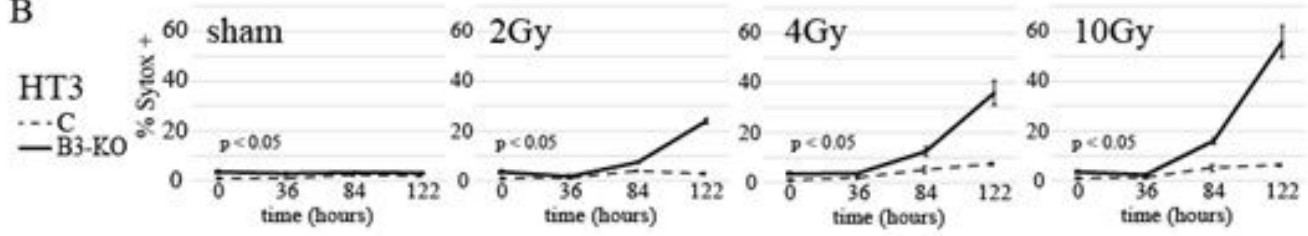

C
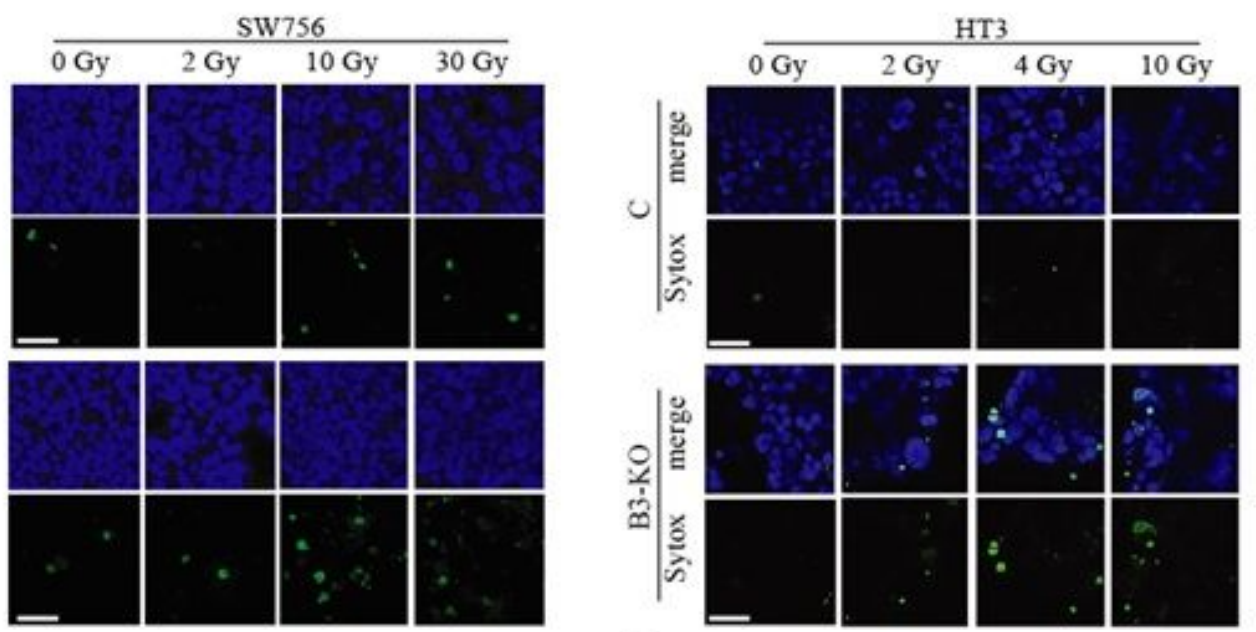

D

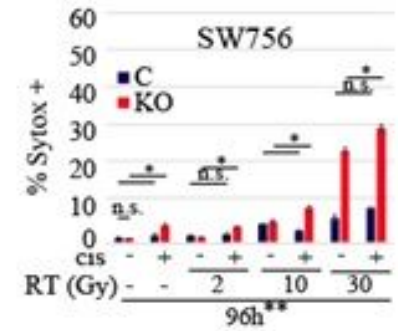

$\mathrm{E}$
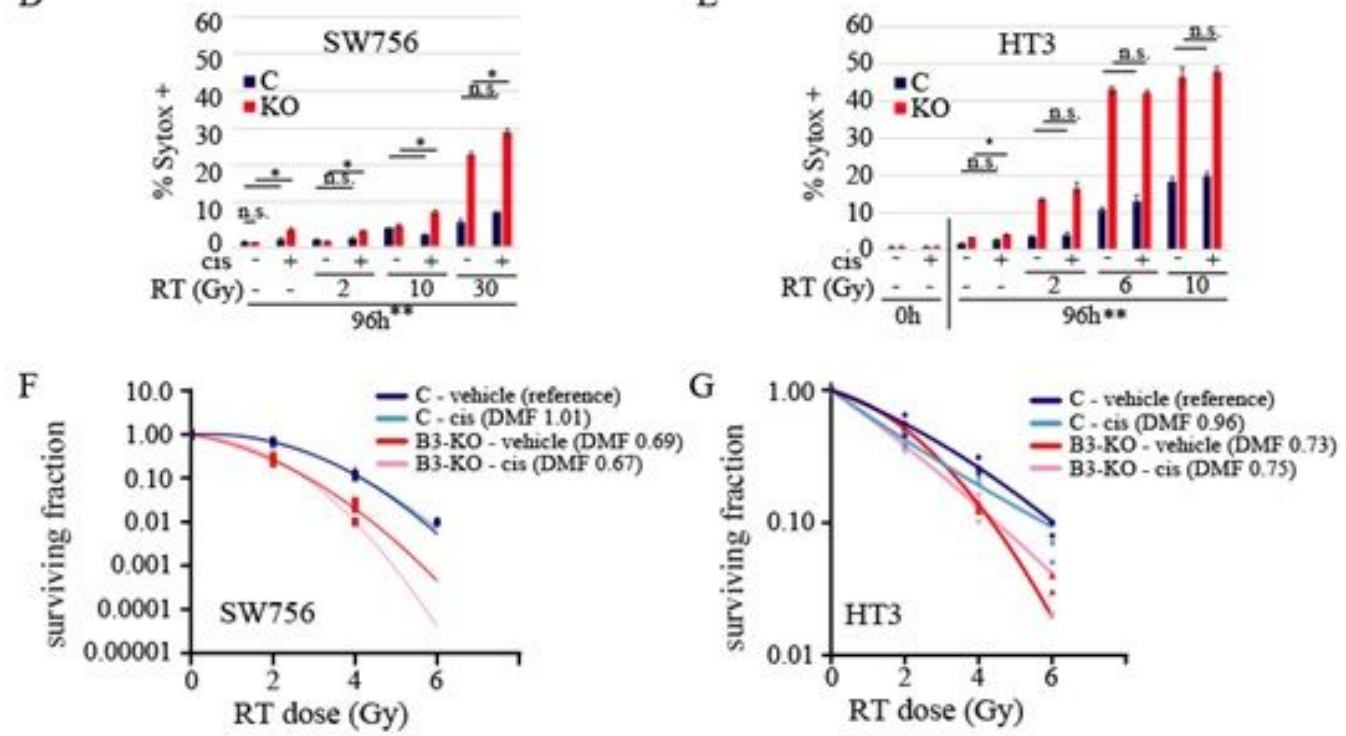

\section{Figure 1}

SERPINB3-KO cells are more sensitive to radiation and cisplatin-induced cell death. A, B) Percent Sytox positive CRISPR-Control (C) and SERPINB3-KO (B3-KO) cells at indicated time points after 2, 10 or 30Gy RT in SW756 (A) and 2, 4 or 10Gy in HT3 (B). Mean and standard deviation are plotted, p-value displays sample variation for two-way ANOVA with replicates over time, n.s. = not significant. C) Representative confocal fluorescent images of SW756- and HT3-C and -B3-KO Sytox (pseudocolored green) and merge 
of Hoescht (pseudocolored blue) and Sytox images at 84 hours post-treatment. Scale bar indicates $50 \mu \mathrm{m}$. D, E) Percent Sytox positive cells following treatment with increasing doses of RT with or without cisplatin at 96 hours in SW756 (D) and HT3 (E) background. Mean and standard deviation is shown, n.s. $=p$-value not significant, $*=p<0.05, * \star=p<0.05$ for all comparisons of $C$ vs B3-KO, except where indicated. F, G) Clonogenic survival of $\mathrm{C}$ or B3-KO cells treated with increasing doses of RT with or without cisplatin in SW756 (F) or HT3 (G) background. Individual data-points from a representative experiment with fit linear quadratic equation. DMF for $10 \%$ surviving fraction is shown relative to that of $\mathrm{C}$ cells treated with vehicle (reference).

A

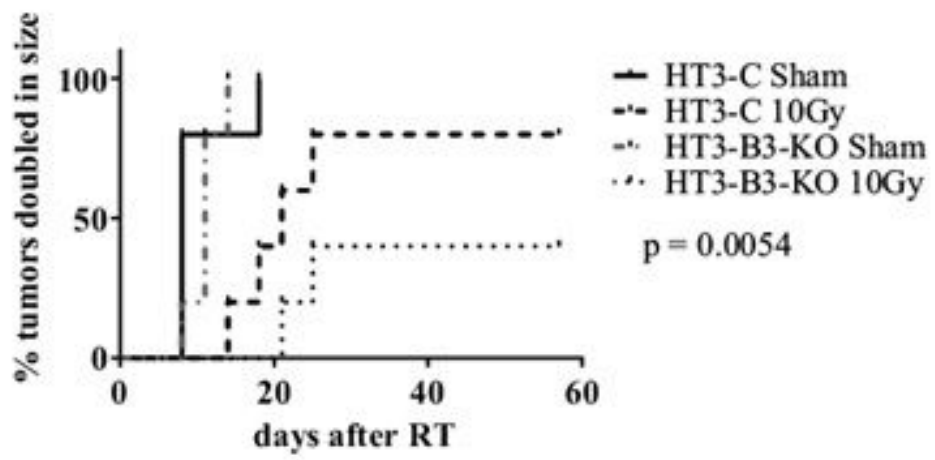

$\mathrm{C}$

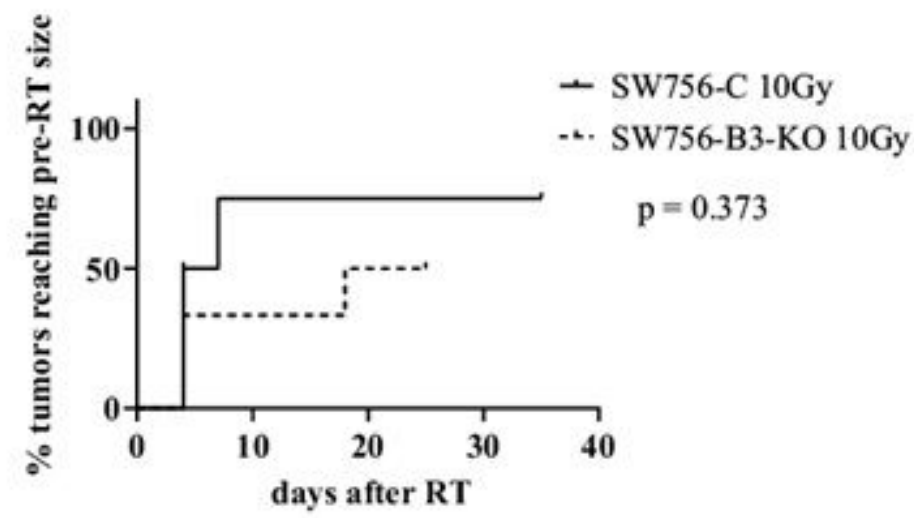

B

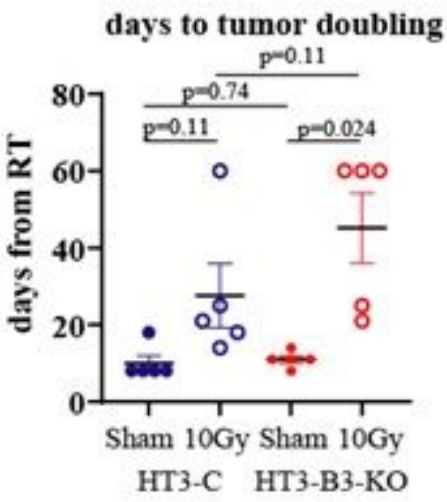

D

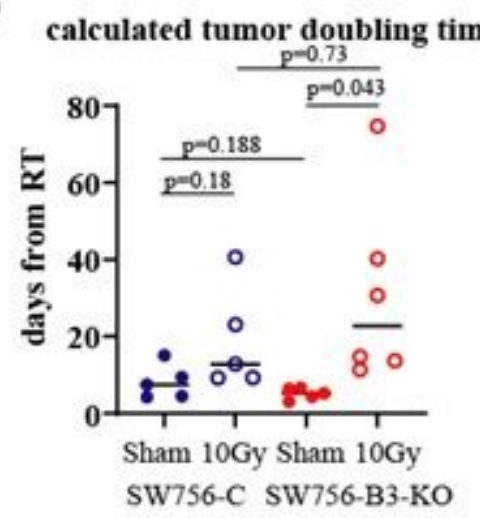

Figure 2

SERPINB3-KO tumors are more sensitive to radiation in vivo. A) Kaplan-Meier curves of percent of HT3 tumors that have doubled in size with log-rank (Mantel-Cox) test $p$-value shown. B) The number of days from RT to tumor doubling is shown for HT3-C and -B3-KO cells after sham or 10Gy. Individual data points with mean and standard error are shown. The four tumors that did not double (one in HT3-C 10Gy group and 3 in B3-KO 10Gy group) were assigned a value of 60 days, at which point the experiment was terminated due to COVID-19-related lab closure. Paired t-test comparisons are shown. C) Kaplan-Meier curves of percent of irradiated SW756 tumors reaching pre-RT size over time. P-value for log-rank statistic is shown. D) Tumor doubling time is plotted for SW756-C and -B3-KO tumors treated with sham or 10Gy RT. As only 2 tumors in the irradiated SW-756-C group and none of the tumors in the SW-756-B3-KO irradiated group doubled in size during the course of the experiment, doubling time was calculated as 
In2/tumor growth rate (TGR) where TGR = \% increase in tumor volume/day. Paired t-test comparisons are shown.

A

HT3

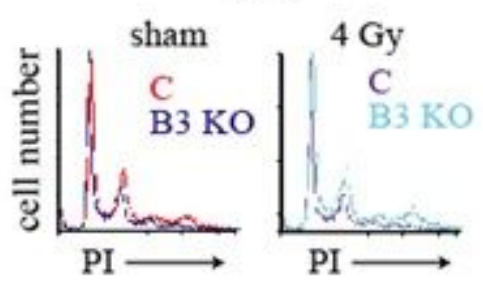

$\mathrm{C}$

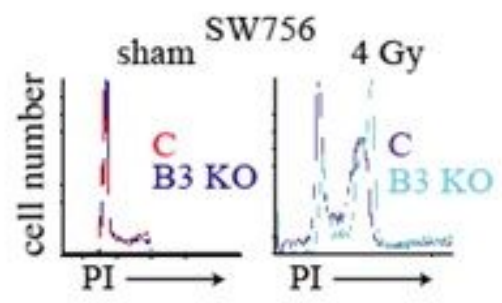

B

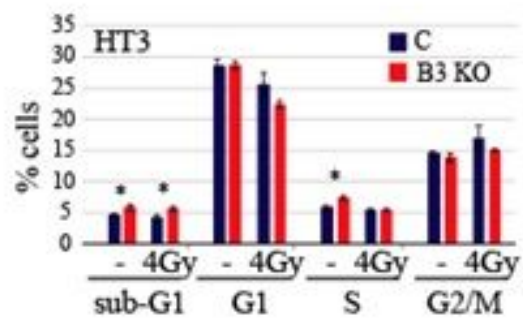

$\mathrm{D}$

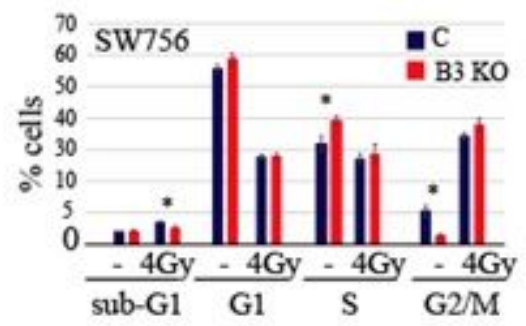

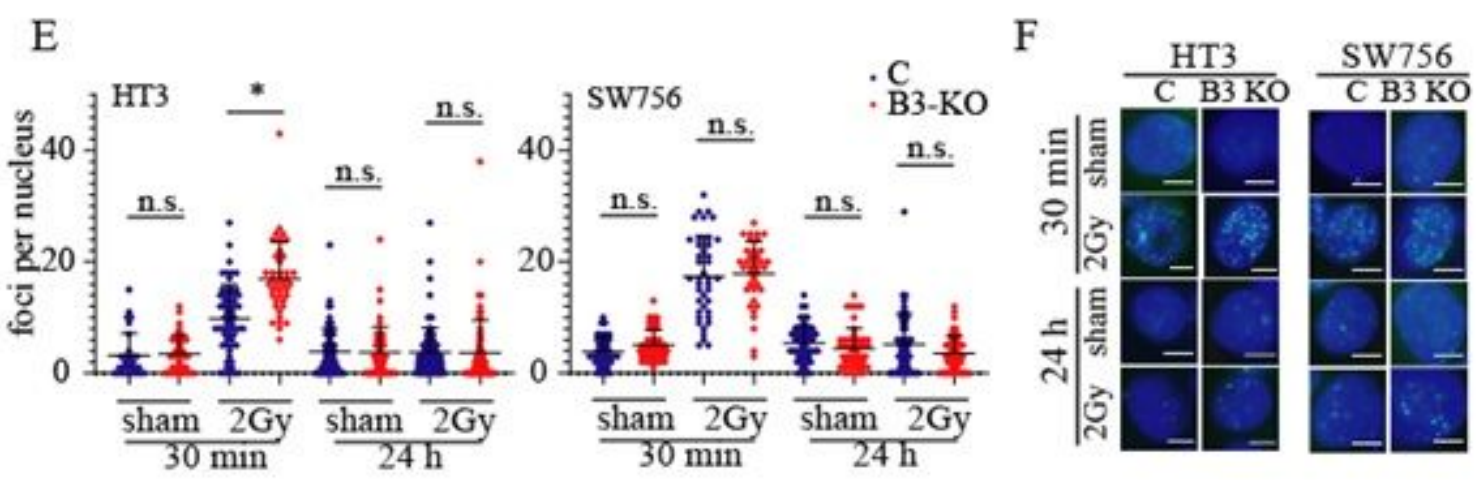

G

HT3

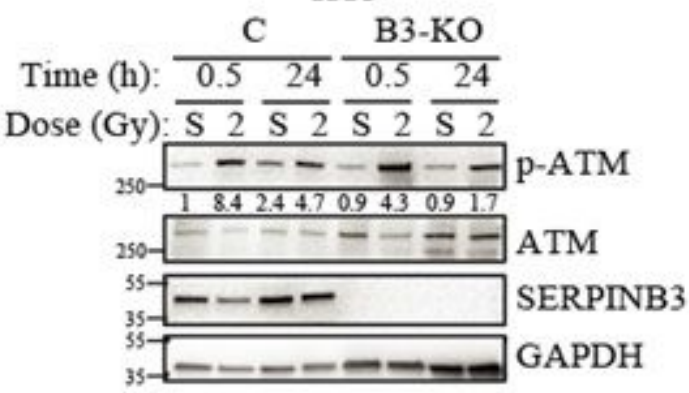

$\mathrm{H}$

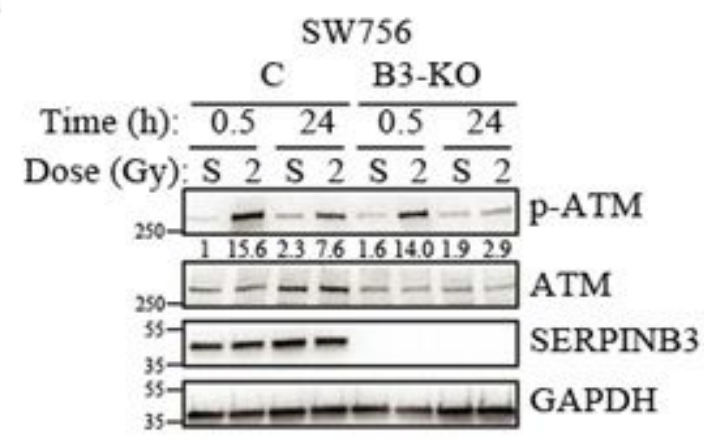

\section{Figure 3}

Increased B3-KO cell death is not explained by cell cycle distribution or impaired DNA damage repair. A, C) Representative histograms for DNA content determined by propidium iodide staining and flow cytometry analysis of HT3 (A) or SW756 (C) cells, with PI intensity on the x-axis. B, D) Mean and standard deviation quantified cell cycle distribution for triplicate repeats of HT3 (B) and SW756 (D) experiments. Asterisks indicate students t-test $p$-value $<0.05$. E) $\otimes H 2 A X$ foci per nucleus in HT3- and SW756-C and -B3-KO cells 
30 minutes or 24 hours after treatment with sham or $2 \mathrm{~Gy}$. Dots represent individual nuclei, with mean and S.D. overlay. N.s. $=$ not significant ${ }^{*}=p<0.05$. F) Representative nuclei shown for each condition with Hoeshct 33342 (blue) and $\triangle \mathrm{H} 2 \mathrm{AX}$ (green) merge. White scale bars shown. G, H) Western blot analysis of total cell lysates from HT3- and SW756-C and -B3-KO cells 30 minutes and 24 hours after treatment with sham (S) or 2Gy radiation. Band intensity of p-ATM normalized to band intensity of GAPDH is displayed under p-ATM blot.

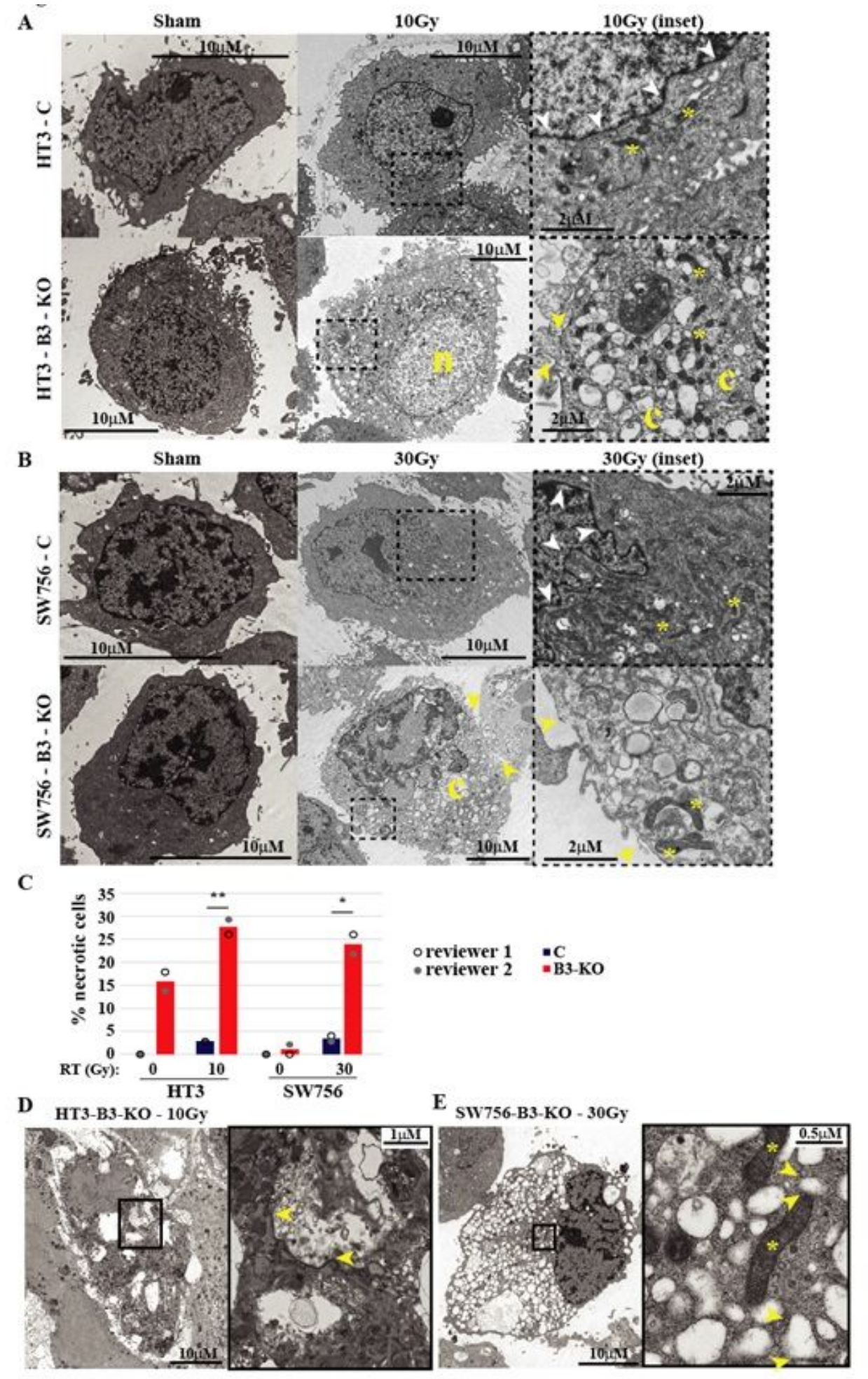


Cell death in B3-KO cells following RT is primarily necrotic. A, B) Transmission electron micrographs of HT3 (A) and SW756 (B) C and B3-KO cells 96 hours after treatment with Sham or indicated doses of RT. Black scale bars shown for each image. Inset shows magnified images of B3-KO irradiated cells and are bound by a dashed black box. Yellow arrows indicate breaks in the plasma membrane, yellow " $\mathrm{c}$ " is cytoplasmic clearing, yellow asterisks indicate mitochondria, and yellow " $n$ " indicates devolving nucleus. C) Quantitation of percent of total cells showing necrotic morphology on 20 random TEM images by two blinded reviewers. Histogram height indicates mean of reviewers, $*=$ student's $t$ test $p$-value $<0.05, * \star=$ student's $t$ test $p$-value $<0.01$. D, E) Representative transmission electron micrographs of HT3-B3-KO (D) and SW756-B3-KO (E) cells 96 hours after treatment with RT, with insets showing high magnification regions delineated by a black box. Cytoplasmic lysosome-like structures with ruptured membranes are indicated by yellow arrows. Mitochondria marked with yellow arrows 
A
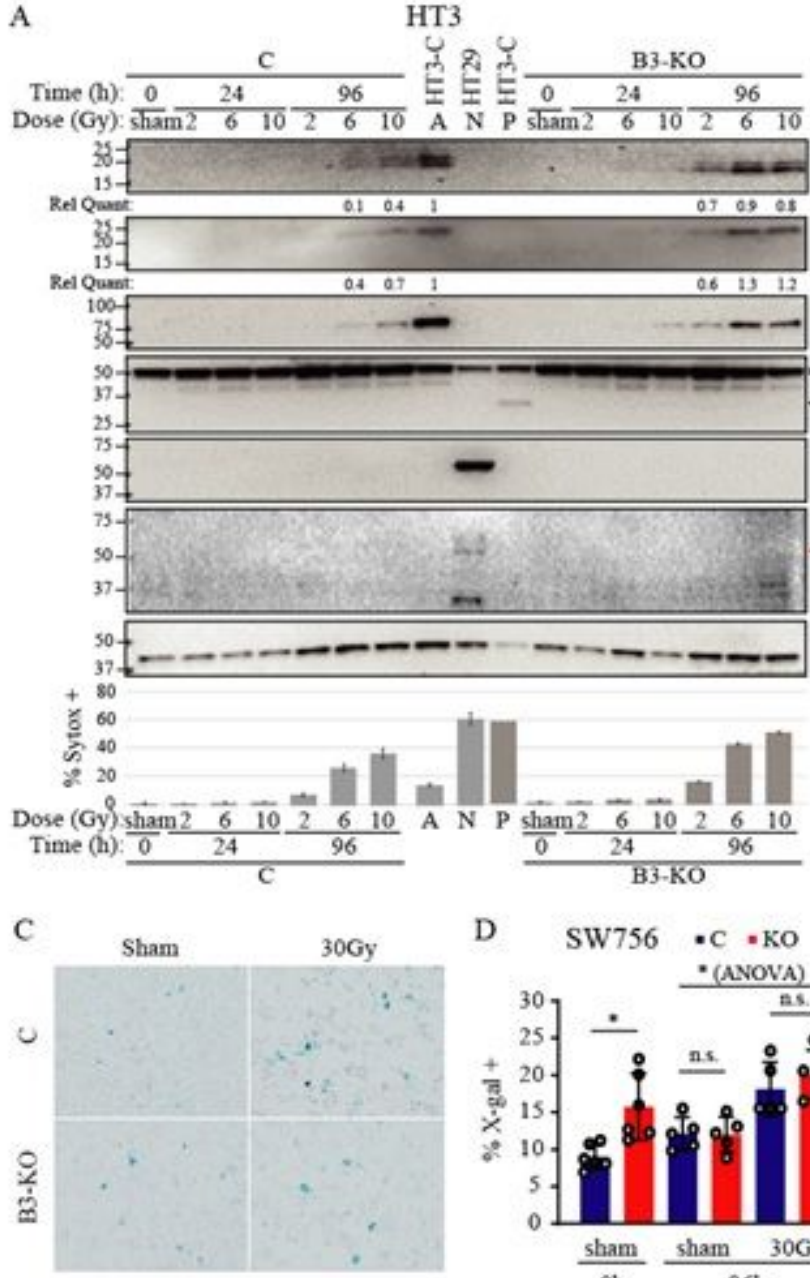

D SW756 $\quad \cdot \mathrm{C} \cdot \mathrm{*}$ KO

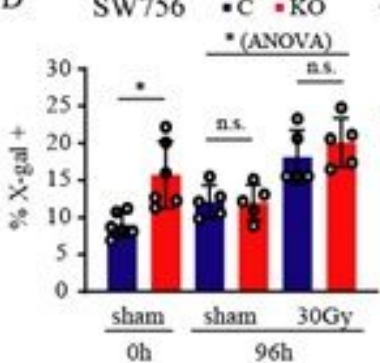

$\mathrm{F}$

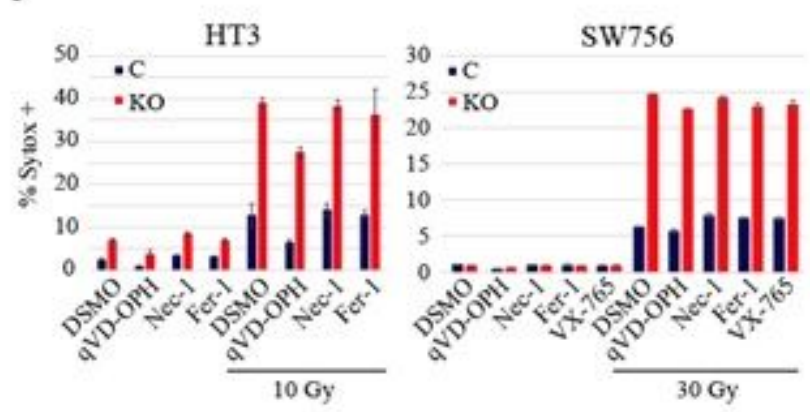

SW756

C
$\frac{0}{0} \frac{24}{24} \frac{96}{5}$
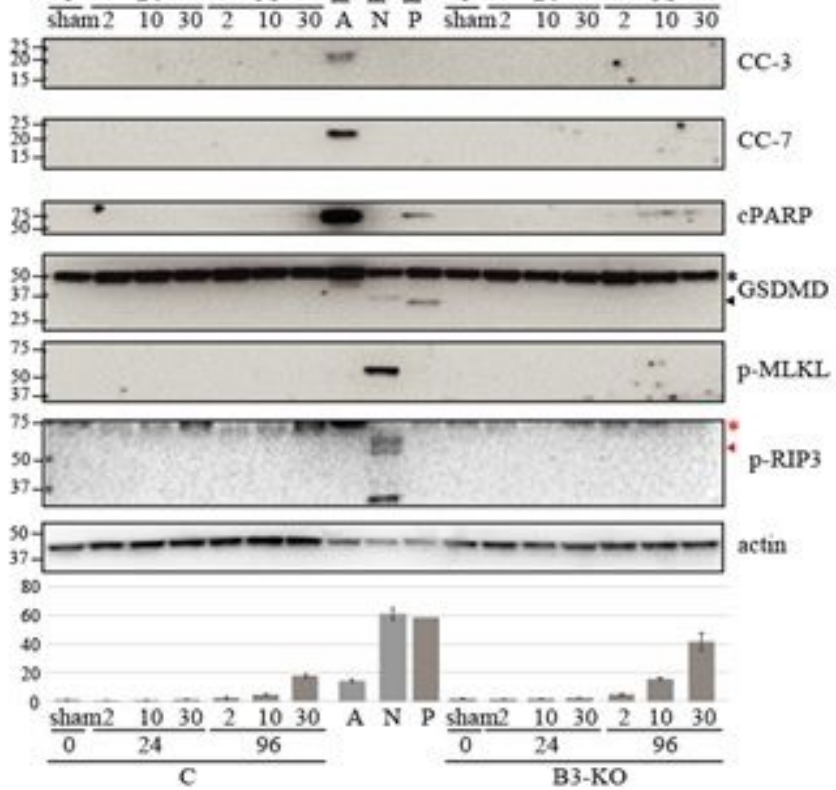

E

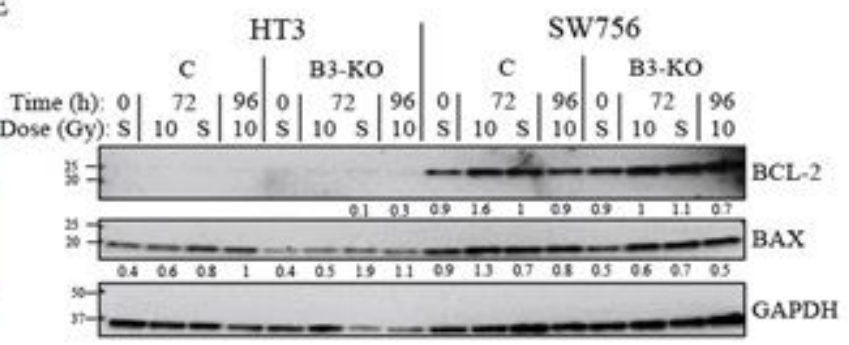

G
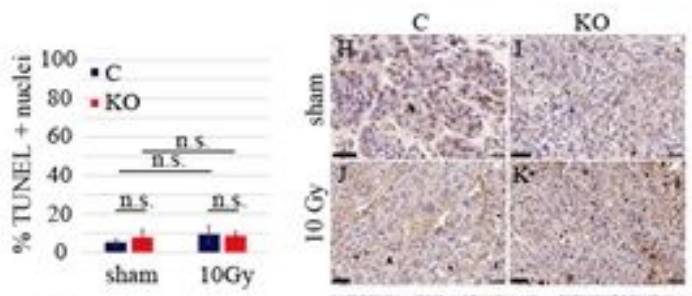

$\mathrm{L}$
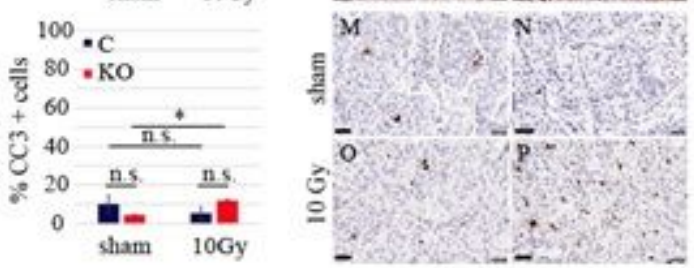

\section{Figure 5}

There is little evidence of apoptosis, necroptosis, ferroptosis, pyroptosis, or senescence in B3-KO cells and tumors treated with ionizing radiation. A) Western blot of total cell lysates from HT3-C, -B3-KO cells at indicated time points following sham or increasing doses of RT. Positive control lysates from HT3-C cells treated with etoposide ("A" for apoptosis), or nucleofected LPS ("P" for pyroptosis), and HT29 cells treated with TNFa, BV6 and ZVAD-fmk ("N" for necroptosis) are shown in the center of the blot. Relative 
intensity of cleaved caspase-3 (CC-3) and cleaved caspase-7 (CC-7) is shown normalized to intensity of actin bands, using the positive control for apoptosis as reference. Histogram beneath shows \% Sytox positive cells for each condition determined simultaneously. Full length GSDMD indicated by black asterisk, and p30 cleavage product indicated by black arrow. Phospho-RIPK3 band ( 60kDa) is indicated by a red arrow, $\sim 75 \mathrm{kDa}$ band on SW756 blot (indicated by red asterisk) is residual PARP antibody signal from previous blotting. B) Western blot of total cell lysates from SW756-C, -B3-KO cells at indicated time points following sham or increasing doses of RT. Positive control lysates from HT3-C cells treated with etoposide ("A" for apoptosis), or nucleofected LPS ("P" for pyroptosis), and HT29 cells treated with TNFa, BV6 and ZVAD-fmk ("N" for necroptosis) are shown in the center of the blot. These positive control lysates were generated concurrently with the experiment. Histogram beneath shows \% Sytox positive cells for each condition determined simultaneously. C) Representative light field images of SA-bgal stained SW756-C or B3-KO cells 96 hours after treatment with sham or 30Gy. D) Quantitation of percent positive $\mathrm{X}$-gal staining cells with individual data points, mean and standard deviation. Statistical tests are t-test comparisons except ANOVA as noted, ${ }^{*}=p<0.05$, n.s. $=$ not significant. E) WB of total cell lysates from HT3-C or -B3-KO or SW756-C or -B3-KO cells treated as indicated. Blots were probed with anti-BCL-2, anti$B A X$ and GAPDH as a loading control, with quantified band intensity below, normalized to GAPDH. F) Percent Sytox positive HT3-C or -B3-KO cells (left) or SW756-C or -B3-KO cells (right) treated with indicated pharmacologic inhibitors 24 hours after sham or radiation. G) Percent TUNEL + nuclei / total nuclei determined on TUNEL stained formalin fixed paraffin embedded sections from indicated tumors. Mean and standard error of the mean are shown, n.s. = not significant. $\mathrm{H}-\mathrm{K})$ representative images of TUNEL staining. Scale bar $=40 \mu \mathrm{m}$. L) Histogram of percent cleaved caspase-3 (CC3) + nuclei / total nuclei determined on $\mathrm{IHC}$ with $\mathrm{CC} 3$ antibody stained from indicated tumors. Mean and standard error of the mean are shown, ${ }^{*}=p<0.05$, n.s. $=$ not significant. $\left.M-P\right)$ representative images of CC3 staining. Scale bar $=40 \mu \mathrm{m}$. 

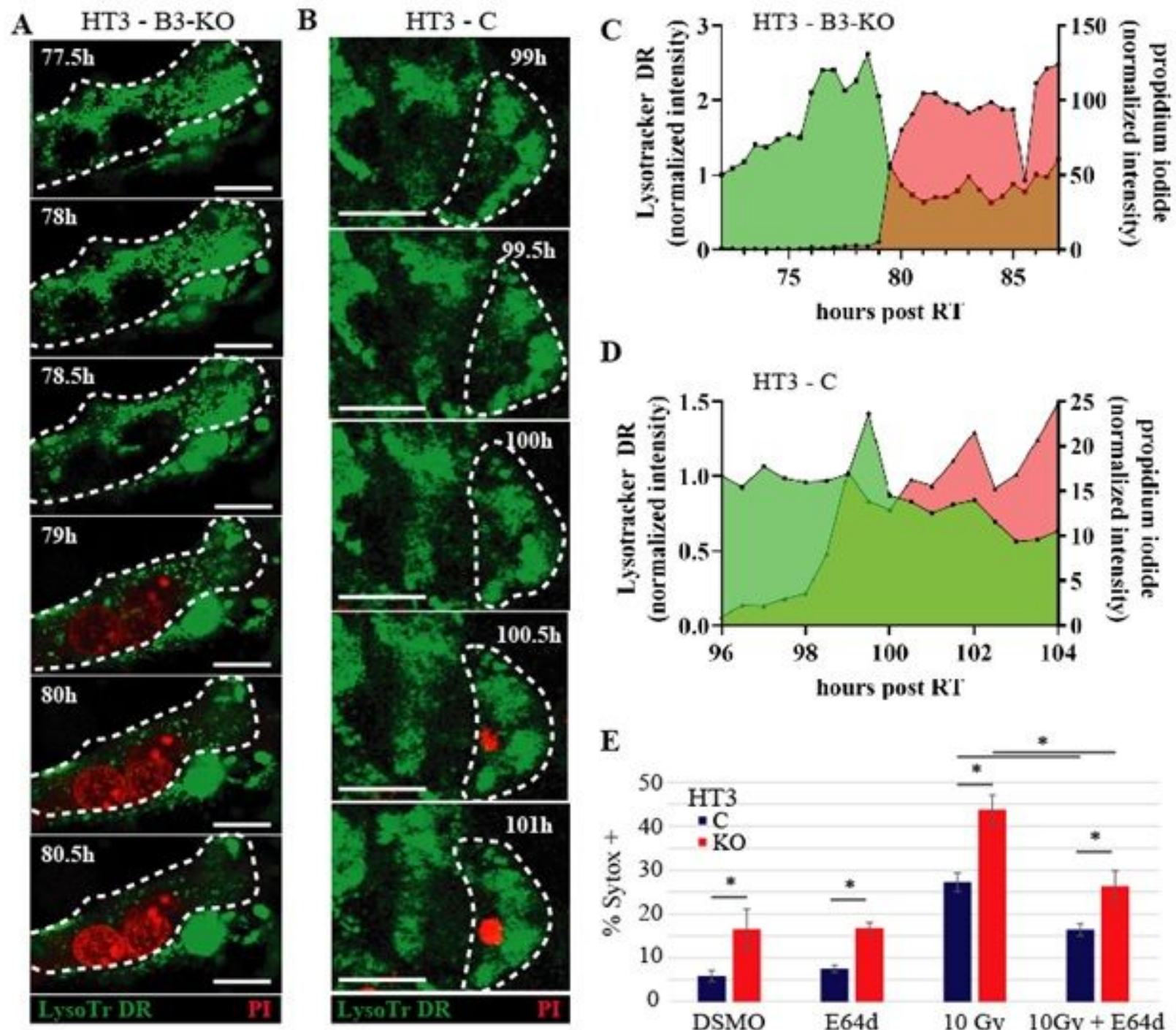

D
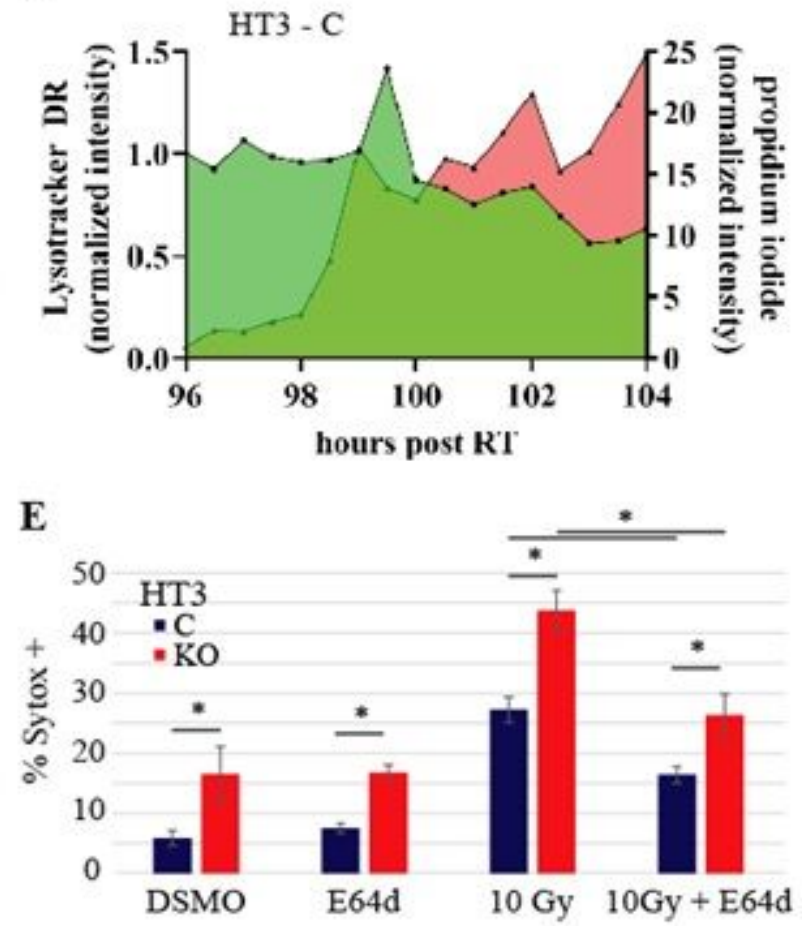

\section{Figure 6}

Radiation induces loss of lysosomal membrane integrity that precedes cell death. A, B) Live cell timelapse confocal pseudocolor images of HT3-B3-KO (A) and HT3-C (B) cells at the indicated times after treatment with 10Gy RT. LysoTracker deep red is pseudocolored green and propidium iodide is pseudocolored red. Scale bar $=25 \mathrm{~mm}$. C, D) cells shown were manually tracked over the timeframe indicated on the x-axis and intensity of LysoTracker deep red (LysoTr) and propidium iodide normalized to cell volume is plotted on the double y-axis. E) Percent Sytox positive HT3-C and -B3-KO cells treated with vehicle DMSO or E64d 24 hours after sham or 10Gy radiation. * $=p<0.05$ by double-sided t-test. 
A

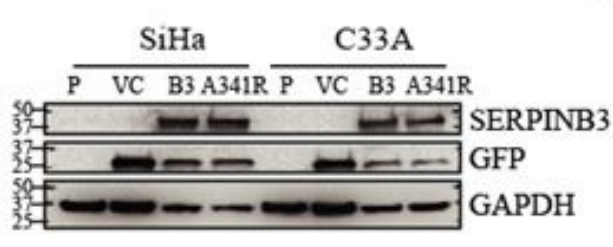

C

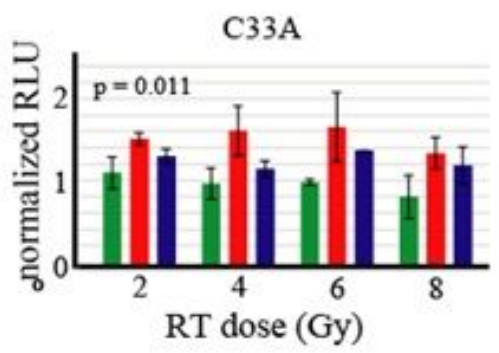

D

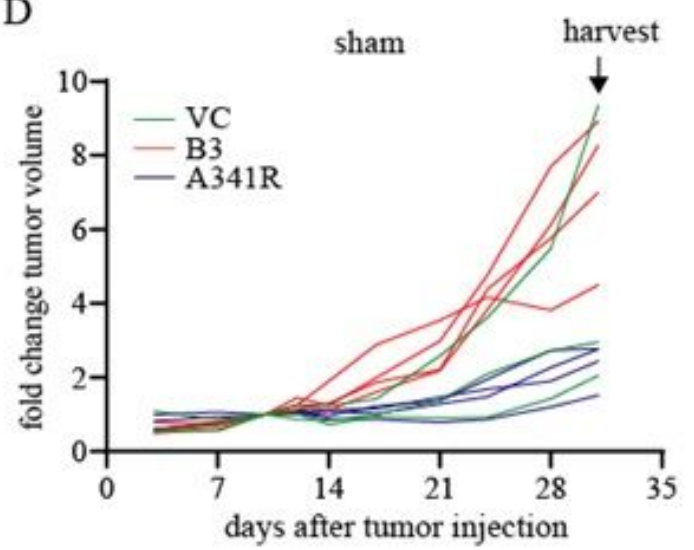

F

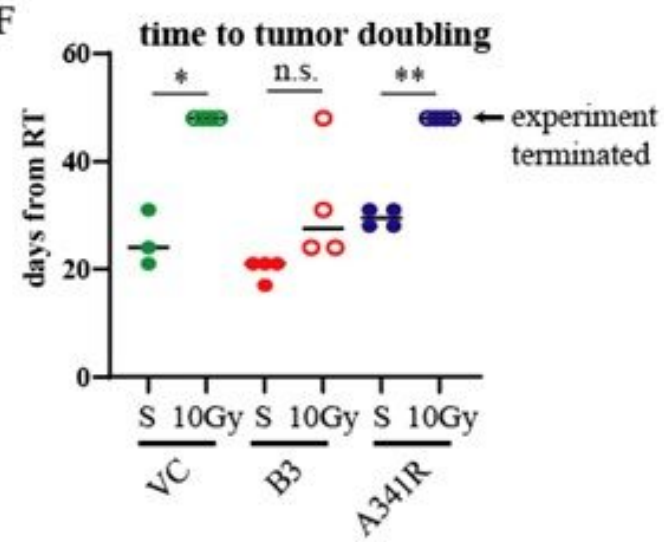

B
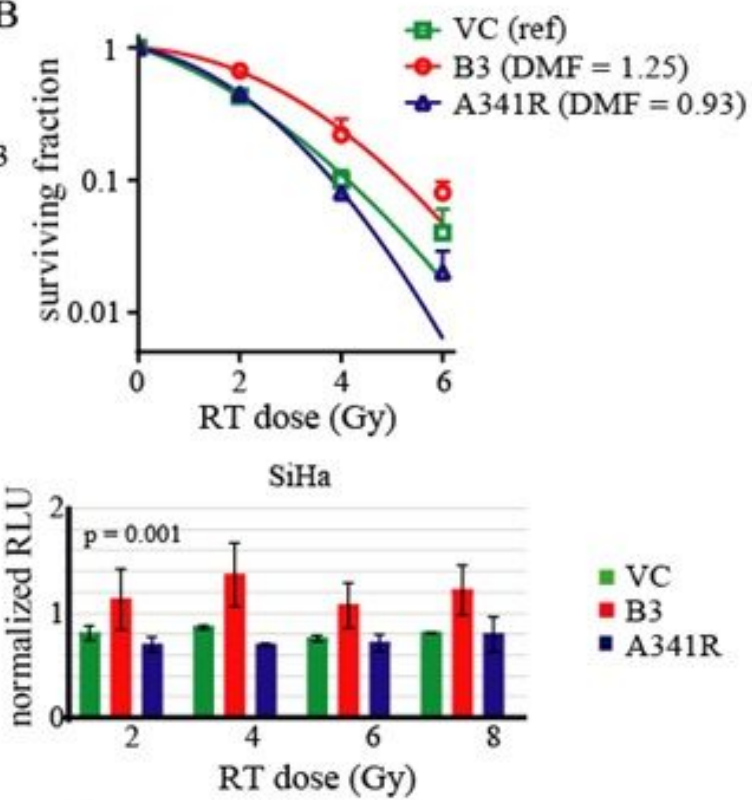

E

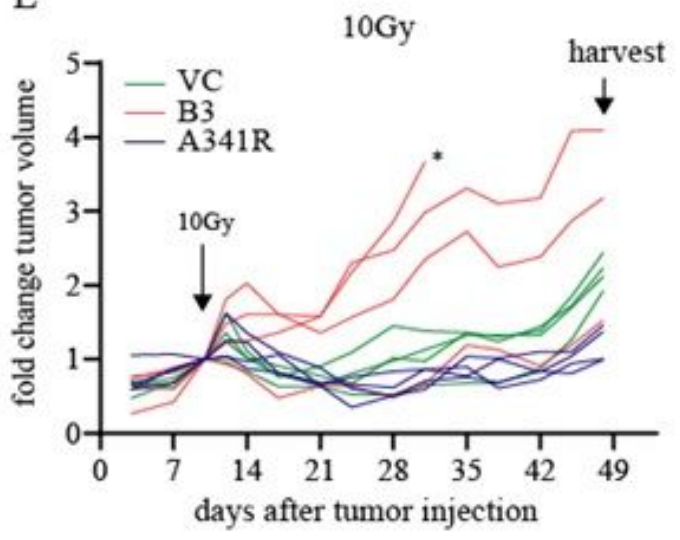

G

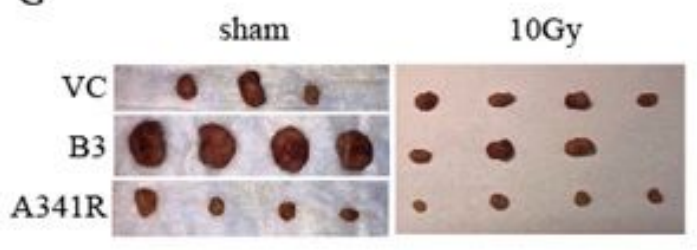

\section{Figure 7}

SERPINB3 is sufficient to protect cervical cancer cells against radiation-induced death, but requires a functional reactive site loop. A) Western blot of SiHa and C33A parental cervical tumor cell lines (P) which do not express SERPINB3 protein at baseline, and stable clones engineered to express wild-type SERPINB3 (B3), SERPINB3-A341R mutant (A341R), or empty vector control (VC). B) Clonogenic survival of SiHa cells expressing VC, B3 or B3-A341R constructs, following increasing doses of RT. C) Relative 
proliferation of C33A cells expressing VC, B3 or B3-A341R treated with RT normalized to Sham-treated cells at 24 hours post-treatment, and measured by relative light units (RLU) of CellTiter-Glo luminescent reagent measuring cellular ATP. Mean and standard deviations are plotted. P-value for one-way ANOVA shown. D, E) Spider plots of individual tumor volume curves of flank xenograft generated from C33A cells expressing VC, B3-wt or B3-A341R treated with sham (D) or 10Gy tumor-directed radiation (E) with tumors harvested at indicated time post-treatment. F) Time to tumor doubling in days for each of the tumors. Individual datapoints are shown, tumors not doubling by the termination of the experiment were included as a data-point on the last day of the experiment. P-value for paired t-test between sham and 10Gy groups for each tumor type are shown. G) Harvested tumors from sham (left) or 10Gy (right) irradiated tumors from experiment shown above.

\section{Supplementary Files}

This is a list of supplementary files associated with this preprint. Click to download.

- SupplementalFigureLegendswithembeddedfigures.docx 